\title{
tramME: Mixed-Effects Transformation Models Using Template Model Builder
}

by Bálint Tamási and Torsten Hothorn

\begin{abstract}
Linear transformation models constitute a general family of parametric regression models for discrete and continuous responses. To accommodate correlated responses, the model is extended by incorporating mixed effects. This article presents the R package tramME, which builds on existing implementations of transformation models (mlt and tram packages) as well as Laplace approximation and automatic differentiation (using the TMB package), to calculate estimates and perform likelihood inference in mixed-effects transformation models. The resulting framework can be readily applied to a wide range of regression problems with grouped data structures.
\end{abstract}

\section{Introduction}

Datasets with grouped observations are abundant in the applied statistical practice. Clustering, hierarchical designs, longitudinal studies, or repeated measurements can all lead to grouped data structures. The common property of these datasets is that observations within groups, defined by one or more grouping factors, cannot be treated as independent. In order to draw a valid inference, the statistical model has to address the issue of correlated observations. Mixed-effects models represent one of the main approaches dealing with this type of regression problem. In this approach, the observations are assumed to be independent conditionally on a set of random effects that aim to capture unmodeled group-level heterogeneity. The reader is referred, for example, to the textbook by Demidenko (2013) for an exposition and examples of the usage of mixed-effects models. Several $\mathrm{R}$ packages exist that implement mixed-effects models for specific types of regression problems. The two most notable examples are nlme by Pinheiro et al. (2021) and lme4 by Bates et al. (2015) for linear, non-linear, and generalized linear mixed-effects models, respectively.

Linear transformation models aim to directly specify the conditional distribution function of an outcome variable in a regression setting. Hothorn (2020) proposed a fully parametric approach using a flexible monotone increasing transformation function that is estimated from the data. The resulting general model family can be applied to a wide range of problems with at least ordered discrete outcome variables. In fact, many of the popular regression models can be expressed as special cases of the linear transformation model framework. Most recently, Tian et al. (2020) reviewed the approach followed in this study and compared it to an alternative semiparametric formulation using extensive simulations. By introducing random effects in the linear transformation model, it becomes applicable in a very diverse set of regression problems where the observations are correlated due to repeated measurements or grouped designs.

The structure of this article is as follows: After a brief, and somewhat technical, introduction of the methodology and the implementation in Section 2.2, Section 2.3 demonstrates, through a series of examples, how the package tramME (Tamási and Hothorn, 2021) can be applied to estimate regression models with various response types and data structures. Finally, Section 2.4 discusses a few issues concerning the implementation of our model.

\section{Mixed-effects transformation models}

The model class in the $\mathrm{R}$ package tramME is an extension of the transformation model approach described by Hothorn et al. (2018) and implemented in the R packages mlt and tram by Hothorn (2020) and Hothorn and Barbanti (2021), respectively. These resources provide an introduction to fully parameterized transformation models for independent observations.

Formally, we are interested in models that parameterize the conditional distribution function directly,

$$
\mathbb{P}(Y \leq y \mid \mathbf{x}, \mathbf{u}, \boldsymbol{\gamma})=F_{Z}\left(h(y ; \boldsymbol{\vartheta})-\mathbf{x}^{\top} \boldsymbol{\beta}-\mathbf{u}^{\top} \boldsymbol{\gamma}\right) \quad \gamma \sim \mathcal{N}_{q}(\mathbf{0}, \boldsymbol{\Sigma}),
$$

where $F_{Z}$ denotes a pre-specified error distribution function (or inverse-link function), which is monotone increasing and maps from the real numbers to the closed interval $[0,1]$. Typically, $F_{Z}$ is set to the CDF of a simple continuous distribution, hence the name "error distribution". The baseline transformation function is $h(y ; \vartheta)$, which is also a monotonic increasing function parameterized with the vector $\vartheta$. For the fixed and random effects design matrices, respectively, $\mathbf{x}^{\top}$ and $\mathbf{u}^{\top}$ are suitable row 
vectors. The vector $\beta$ contains the fixed effects, while $\gamma$ comprises of the stacked (possibly multiple) random effects. The distribution of the random effects is assumed to be multivariate Gaussian with zero mean and covariance matrix $\Sigma$, which typically has a sparse block structure.

As Table 1 shows, specific choices of the error distribution and the baseline transformation function lead to different types of regression models. In the $\mathrm{R}$ package tramME, seven main model types are distinguished, mainly based on the class of their outcome variable. Moreover, the functions SurvregME() and PolrME() allow to specify multiple error distributions or baseline transformations and hence increasing the number of available model types.

\begin{tabular}{|c|c|c|c|}
\hline Function & Name & $F_{Z}$ & $h(y ; \vartheta)$ \\
\hline $\operatorname{LmME}()$ & $\begin{array}{l}\text { Mixed-effects normal linear } \\
\text { regression }\end{array}$ & Standard Gaussian & Linear basis \\
\hline BoxCoxME() & $\begin{array}{l}\text { Non-normal (Box-Cox-type) } \\
\text { linear mixed-effects regression }\end{array}$ & Standard Gaussian & Bernstein basis \\
\hline Colrme() & $\begin{array}{l}\text { Mixed-effects continuous } \\
\text { outcome logistic regression }\end{array}$ & Standard logistic & Bernstein basis \\
\hline CoxphME() & $\begin{array}{l}\text { Mixed-effects parametric Cox } \\
\text { regression }\end{array}$ & Minimum extreme value & Bernstein basis \\
\hline SurvregME() & $\begin{array}{l}\text { Mixed-effects parametric } \\
\text { survival models }\end{array}$ & Multiple options & Multiple options \\
\hline Polrme() & $\begin{array}{l}\text { Mixed-effects regression models } \\
\text { for ordinal outcomes }\end{array}$ & Multiple options & Discrete basis \\
\hline LehmannME() & $\begin{array}{l}\text { Mixed-effects } \\
\text { Lehmann-alternative linear } \\
\text { regression }\end{array}$ & Maximum extreme value & Bernstein basis \\
\hline
\end{tabular}

Table 1: Model types implemented in the tramME package. $F_{Z}$ denotes the error distribution and the column $h(y ; \vartheta)$ lists the basis functions the baseline transformation function utilizes.

As the table indicates, some of the models specify their transformation functions as general smooth functions, approximated with the use of polynomials in Bernstein form. The function $h(y ; \vartheta)$ has to be monotonic increasing so that the conditional distribution function is also increasing. When using a set of order $p$ polynomials in Bernstein form for the approximation of a general function, this restriction conveniently translates to the parameter restriction $\vartheta_{i} \leq \vartheta_{i+1}$ for all $i=0, \ldots, p-1$.

The observations are assumed to be conditionally independent, and hence the likelihood has the form

$$
\begin{aligned}
\mathcal{L}(\boldsymbol{\vartheta}, \boldsymbol{\beta}, \boldsymbol{\Sigma}) & =\int_{\mathbb{R}^{q}} \mathcal{L}(\boldsymbol{\vartheta}, \boldsymbol{\beta}, \boldsymbol{\Sigma}, \boldsymbol{\gamma}) \mathrm{d} \boldsymbol{\gamma} \\
& =\int_{\mathbb{R}^{q}} \prod_{i=1}^{n} \mathcal{L}_{i}(\boldsymbol{\vartheta}, \boldsymbol{\beta} \mid \boldsymbol{\gamma}) \phi(\boldsymbol{\gamma} ; \boldsymbol{\Sigma}) \mathrm{d} \boldsymbol{\gamma}
\end{aligned}
$$

where $\mathcal{L}(\boldsymbol{\vartheta}, \boldsymbol{\beta}, \boldsymbol{\Sigma}, \gamma)$ is the joint likelihood function, given the all observations, and $\mathcal{L}_{i}(\boldsymbol{\vartheta}, \boldsymbol{\beta} \mid \gamma)$ denotes the individual conditional likelihood contributions. $\phi(\gamma ; \Sigma)$ stands for the probability density function of the multivariate normal distribution with zero mean vector and covariance matrix $\Sigma$. This latter function can be factorized further according to the covariance structure of the random effects.

One of the main advantages of working directly with the distribution function of the outcome is that it is simple to introduce (random) censoring and truncation in the estimation procedure. The conditional likelihood contributions under different types of censoring can be written as

$$
\mathcal{L}_{i}(\boldsymbol{\vartheta}, \boldsymbol{\beta} \mid \boldsymbol{\gamma})=\left\{\begin{array}{lll}
f_{Z}\left(h(y ; \boldsymbol{\vartheta})-\mathbf{x}^{\top} \boldsymbol{\beta}-\mathbf{u}^{\top} \boldsymbol{\gamma}\right) h^{\prime}(y ; \boldsymbol{\vartheta}) & y \in \Xi & \text { "exact continuous" } \\
1-F_{Z}\left(h(\underline{y} ; \boldsymbol{\vartheta})-\mathbf{x}^{\top} \boldsymbol{\beta}-\mathbf{u}^{\top} \boldsymbol{\gamma}\right) & y \in(\underline{y}, \infty) \cap \Xi & \text { "right-censored" } \\
F_{Z}\left(h(\bar{y} ; \boldsymbol{\vartheta})-\mathbf{x}^{\top} \boldsymbol{\beta}-\mathbf{u}^{\top} \boldsymbol{\gamma}\right) & y \in(-\infty, \bar{y}] \cap \Xi & \text { "left-censored" } \\
F_{Z}\left(h(\bar{y} ; \boldsymbol{\vartheta})-\mathbf{x}^{\top} \boldsymbol{\beta}-\mathbf{u}^{\top} \boldsymbol{\gamma}\right) & \\
-F_{Z}\left(h(\underline{y} ; \boldsymbol{\vartheta})-\mathbf{x}^{\top} \boldsymbol{\beta}-\mathbf{u}^{\top} \boldsymbol{\gamma}\right) & y \in(\underline{y}, \bar{y}] \cap \Xi & \text { "interval-censored", }
\end{array}\right.
$$


where $f_{Z}()$ is the density function of the error distribution, $h^{\prime}(y ; \vartheta)$ is the first derivative of the baseline transformation function with respect to $y$, and $\Xi$ denotes the sample space of $Y$.

The multidimensional integral in Equation (2), in general, does not have an analytical solution, but its value can be approximated using numerical methods. The package tramME applies the Laplace approximation to this problem, which relies on the quadratic Taylor expansion of the corresponding joint log-likelihood function.

The maximization of the logarithm of the likelihood function with respect to $\boldsymbol{\vartheta}, \boldsymbol{\beta}$, and $\boldsymbol{\Sigma}$, under a set of suitable constraints on $\vartheta$ to make $h(y ; \vartheta)$ monotone increasing, results in the maximum likelihood estimates of the model parameters. Standard likelihood theory, utilizing the ability to evaluate the log-likelihood function, the score function, and the Hessian, provides a basis for asymptotic inference in this family of models; see Hothorn et al. (2018) for more details on likelihood inference in transformation models.

The maximum likelihood estimation in tramME is done using the TMB package by Kristensen et al. (2016). The Template Model Builder (TMB) allows the user to define and estimate general, non-linear mixed-effects models. It was built on well-tested and high-performance $\mathrm{C}++$ libraries, which results in a flexible yet efficient framework for estimating mixed models with possibly complex random effects structures; see, for example, Brooks et al. (2017) for performance comparisons in the context of the package glmm TMB. In tramME, TMB is used to evaluate the integral in Equation (2), using Laplace's method, and to calculate the derivatives of the log-likelihood function using automatic (or algorithmic) differentiation.

\section{Applications}

In this section, several applications of the transformation mixed models are presented, and wherever it is possible, also compared to other existing implementations. The examples shown here are by no means intended as complete analyses. They demonstrate how mixed-effects transformation models can be used in a broad range of regression problems and showcase the most important features implemented in the package tramME.

In each application, a simple version of a transformation mixed model is compared to the same model implemented by a benchmark package first. In a second step, extensions to more complex models not available other packages are fitted using package tramME. The R code illustrates similarities and differences in the user interfaces. The two model outputs allow a direct comparison of the modelagnostic implementation in tramME to the model-specific implementation in the benchmark package. The package tramME is, however, not intended as a replacement for well-tested implementations of important special cases of mixed models, such as linear mixed models in 1me 4 , but as a tool for extending these implementations to more complex model variants.

\section{Normal linear mixed model}

As a first example, we model the average reaction times to a specific task from a sleep deprivation study described in Belenky et al. (2003). Figure 1 presents the reaction times against days of sleep deprivation for each of the 18 participants.

In this first example, we model the distribution of the average reaction time using random intercepts and random slopes for the effects of days of sleep deprivation.

$$
\begin{aligned}
\mathbb{P}\left(\text { Reaction } \leq y \mid \text { Days, } \alpha_{i}, \beta_{i}\right) & =\Phi\left(\vartheta_{1}+\vartheta_{2} y-\beta \text { Days }-\gamma_{1 i}-\gamma_{2 i} \text { Days }\right) \\
\left(\begin{array}{l}
\gamma_{1 i} \\
\gamma_{2 i}
\end{array}\right) & \sim \mathcal{N}_{2}\left\{\left(\begin{array}{l}
0 \\
0
\end{array}\right),\left(\begin{array}{cc}
\tau_{1}^{2} & \tau_{12} \\
\tau_{12} & \tau_{2}^{2}
\end{array}\right)\right\}
\end{aligned}
$$

Note that when the transformation function is assumed to be linear in the outcome variable, i.e., $h(y)=\vartheta_{1}+\vartheta_{2} y$, we arrive at a re-parameterized version of the normal linear mixed effects-model, and hence the results from tramME : $\operatorname{LmME}()$ are directly comparable to estimates using other mixed-effects regression packages such as lme4. Estimating the normal linear model with the tramME:

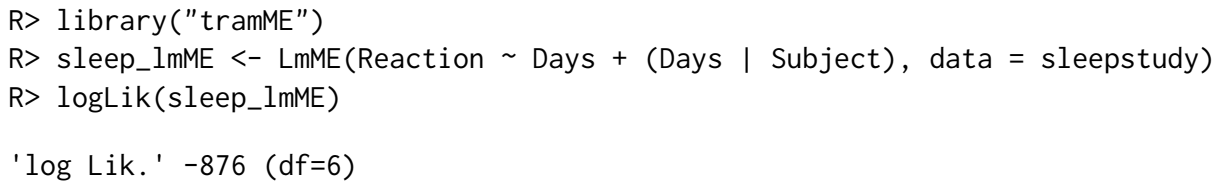

To make the results from lme4 comparable to the previous results, we set REML = FALSE, as the transformation mixed model implementation only supports the maximum likelihood estimation of 


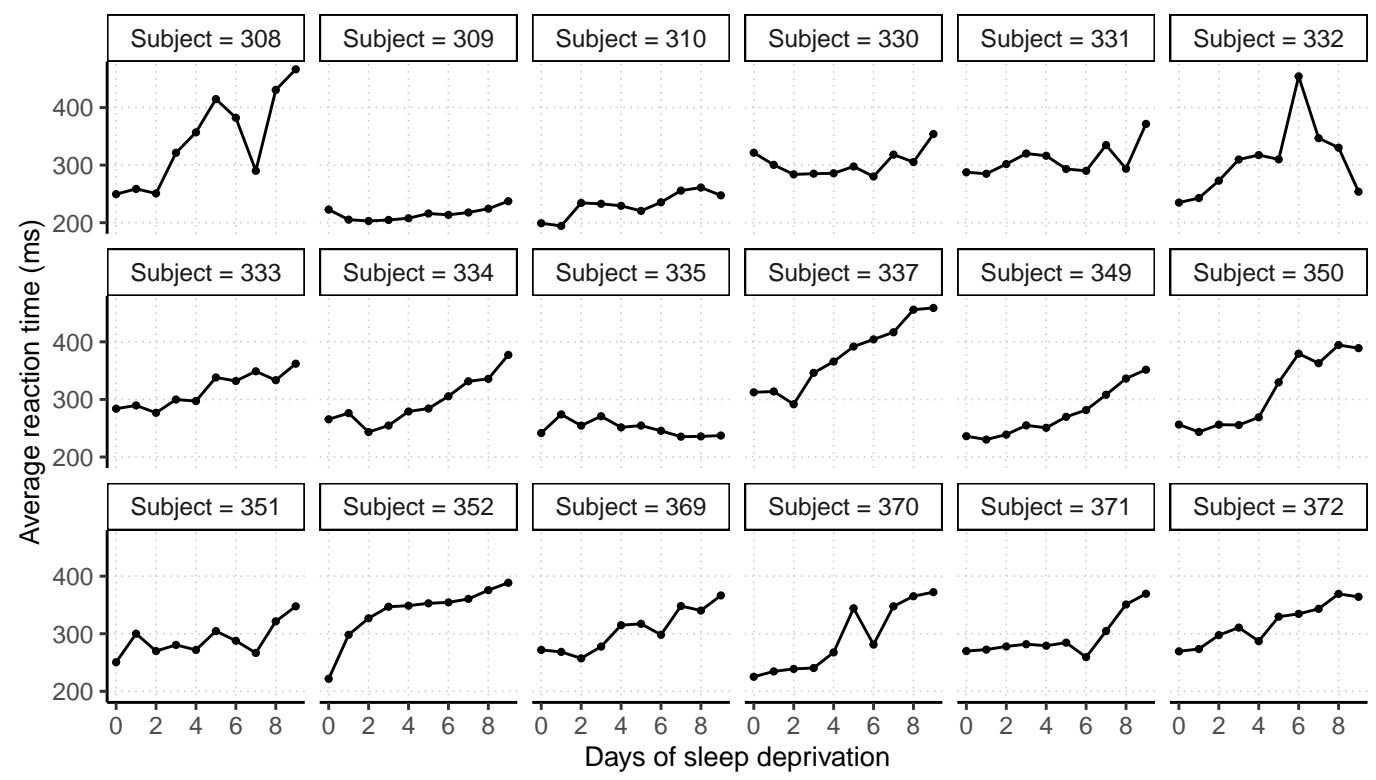

Figure 1: Sleep deprivation study: Average reaction times to a specific task of 18 participants after several days of sleep deprivation reported by Belenky et al. (2003).

the normal linear model specification.

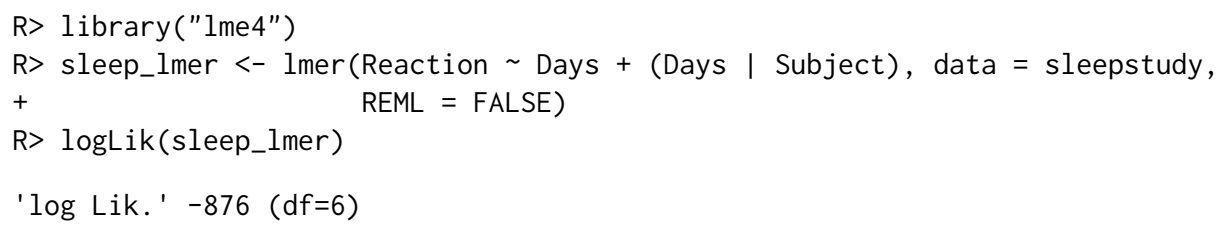

The as. $1 \mathrm{~m}$ = TRUE option of various methods in tramME facilitates the comparisons between the transformation model parameterization and the results of a linear mixed model parameterization. Coefficient estimates and their standard errors from the transformation model approach are

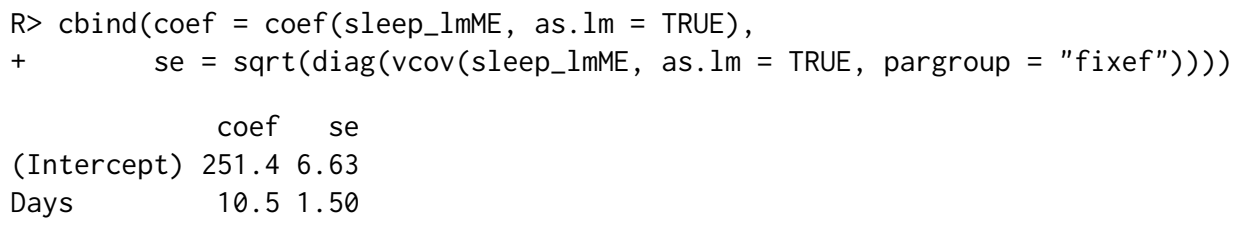

while the results from lmer are

R> summary(sleep_lmer)\$coefficients

$\begin{array}{lrrr} & \text { Estimate } & \text { Std. Error } t & \text { value } \\ \text { (Intercept) } & 251.4 & 6.63 & 37.91 \\ \text { Days } & 10.5 & 1.50 & 6.97\end{array}$

Similarly, the standard deviations and correlations of the random effects and the standard deviations of the error terms are essentially the same

R> VarCorr (sleep_lmME, as.1m = TRUE) \#\# random effects

Grouping factor: Subject (18 levels)

Standard deviation:

$\begin{array}{rr}\text { (Intercept) } & \text { Days } \\ 23.80 & 5.72\end{array}$

Correlations:

(Intercept)

Days $\quad 0.0813$ 
R> sigma(sleep_lmME) \#\# residual SD

[1] 25.6

R> VarCorr(sleep_lmer)

$\begin{array}{llrl}\text { Groups } & \text { Name } & \text { Std.Dev. } & \text { Corr } \\ \text { Subject } & \text { (Intercept) } & 23.78 & \\ & \text { Days } & 5.72 & 0.08 \\ \text { Residual } & & 25.59 & \end{array}$

With the predict method of tramME, we can evaluate the fitted conditional distribution of the outcome on a scale specified by the user. Additionally, by setting type = "quantile", we can calculate the quantiles of the conditional distribution of the response.

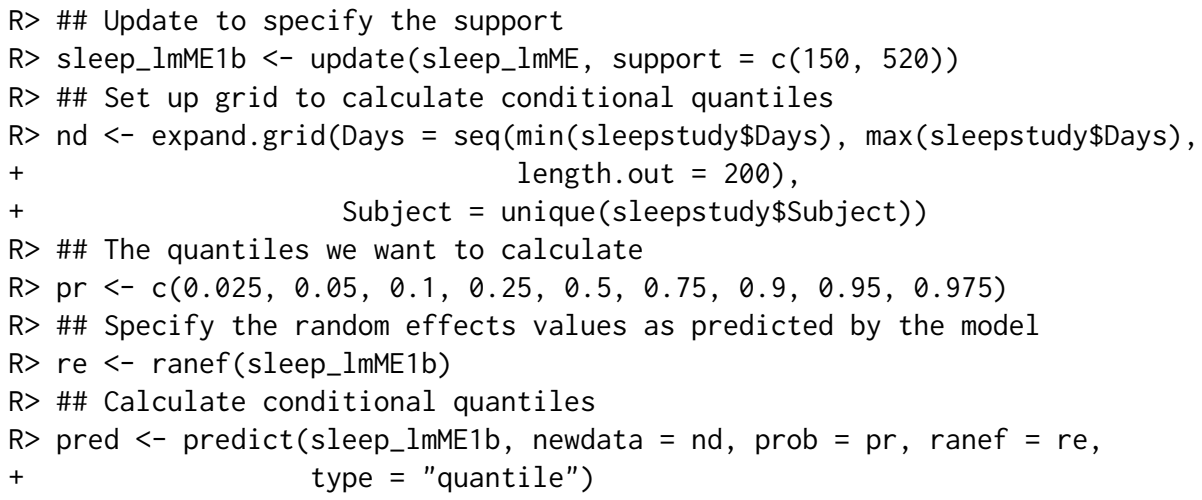

Note that in the code above, we first update the model and explicitly set the support of the outcome distribution. It is often helpful to define the support when we want to calculate the quantiles of the outcome distribution because in certain extreme cases, the calculated values may lie outside of the default support and, in these cases, predict. trammE (just as predict.mlt) will return censored values.

Because we are interested in conditional quantiles, we have to specify the values of the random effects on which we want to condition for each subject. In the example above, we simply set the predicted random effects values for the subjects of the sleepstudy dataset. Although it is relatively common in practice, one should be careful with using plug-in estimators of non-linear functions of the random effects (i.e., estimating these functions by evaluating at the point estimates of the random effects) as they can contain substantial bias (Thorson and Kristensen, 2016). To demonstrate how mixed-effects transformation models relax certain assumptions of the normal linear reference model and to showcase the functionality implemented in the tramME package, occasionally, we will rely on these estimators nevertheless.

Figure 2 presents the quantiles of the conditional distribution of reaction time from the model defined in Equation (3). The random intercepts and slopes capture separate time trends for each subject in the study. In the normal linear mixed model (estimated with LmME), the conditional quantiles are parallel lines. We will revisit this example when we relax certain assumptions of this initial model in Section 2.3.2.

tramME implements a version of score residuals that are defined as the score contributions of the individual observations with respect to an additional constant term that is fixed at zero.

$$
\begin{aligned}
\mathbb{P}(Y \leq y \mid \mathbf{x}, \mathbf{u}, \gamma) & =F_{Z}\left(h(y ; \boldsymbol{\vartheta})-\mathbf{x}^{\top} \boldsymbol{\beta}-\mathbf{u}^{\top} \gamma-\alpha_{0}\right) \quad \gamma \sim \mathcal{N}_{q}(\mathbf{0}, \boldsymbol{\Sigma}) \\
r_{i} & =\left.\frac{\partial \ell_{i}\left(\boldsymbol{\vartheta}, \boldsymbol{\beta}, \boldsymbol{\Sigma}, \alpha_{0}\right)}{\partial \alpha_{0}}\right|_{\alpha_{0}=0,}
\end{aligned}
$$

where $\ell_{i}\left(\boldsymbol{\vartheta}, \boldsymbol{\beta}, \boldsymbol{\Sigma}, \alpha_{0}\right)$ is the marginal log-likelihood contribution of observation $i$. It is straightforward to show that, in the case of the normal linear model, this is equal to the response residuals divided by the MLE of the error standard deviation. As previously, the comparison with the parameterization used by $1 \mathrm{me} 4$ is made easy by using the option as. $1 \mathrm{~m}=$ TRUE.

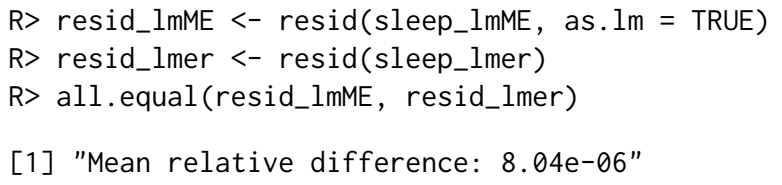

Using the linear predictor of the mixed-effects transformation model, $\left(\mathbf{x}^{\top} \widehat{\boldsymbol{\beta}}+\mathbf{u}^{\top} \widehat{\gamma}\right)$ calculated as 


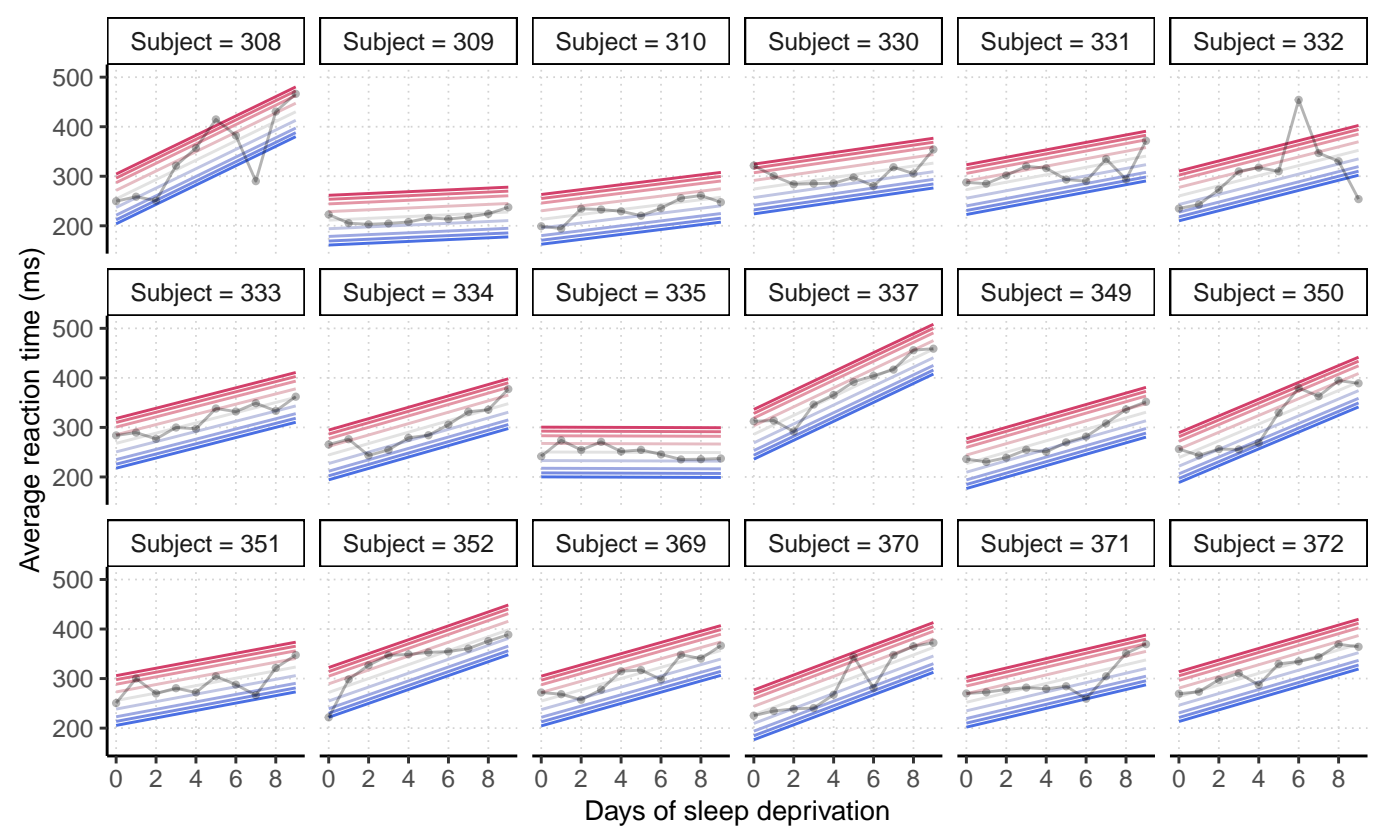

Estimated conditional quantiles

$-0.025-0.05-0.1-0.25-0.5-0.75-0.9-0.95-0.975$

Figure 2: Quantiles of the conditional distribution of the outcome fitted to the sleepstudy data with the normal linear mixed-effects model (LmME).

$\mathrm{R}>1 \mathrm{p}<-$ predict$($ sleep_lmME, type $=$ "lp")

we can construct plots for checking the residuals (Figure 3).

As the results of this section show, the transformation model approach implemented by LmME() leads to the same results as the maximum likelihood estimation of the traditional linear mixed model parameterization. The advantage of using the package tramME over other well-established implementations is that it can also be applied when classical model assumptions are not met. For the sleep deprivation experiments, the data analyst might wonder if assuming normal reaction times is appropriate and if clocking of reaction times was indeed as accurate as suggested by the data (milliseconds with four digits). The former issue requires a relaxation of the conditional normality assumption and the latter incorporation of interval-censoring in the likelihood. We will start with model estimation in the presence of interval-censored reaction times, which is outside the scope of lme4.

Let us assume that the measurement device used in the sleep deprivation study is only able to measure reaction times larger than $200 \mathrm{~ms}$ and only in $50 \mathrm{~ms}$ step sizes. If we want to take this reduced accuracy in the measurements into account, we have to deal with interval-censored observations as ignoring the censored nature of the outcomes could lead to biased parameter estimates.

With the following code, we create the interval-censored outcome vector using the Surv function of the survival package by Therneau (2021).

R> library("survival")

$\mathrm{R}>\mathrm{ub}<-$ ceiling(sleepstudy\$Reaction / 50) *50

$\mathrm{R}>\mathrm{lb}<-$ floor (sleepstudy\$Reaction / 50) * 50

$\mathrm{R}>\mathrm{lb}[\mathrm{ub}==200]<-0$

$\mathrm{R}>$ sleepstudy\$Reaction_ic <- Surv(lb, ub, type = "interval2")

$\mathrm{R}>$ head(sleepstudy\$Reaction_ic)

$[1][200,250][250,300][250,300][300,350][350,400][400,450]$

Using the interval-censored outcomes in the $\operatorname{LmME}($ ), function call will maximize the correct likelihood function.

R> sleep_lmME2 <- LmME(Reaction_ic Days + (Days | Subject), data = sleepstudy)

R> logLik(sleep_lmME2) 

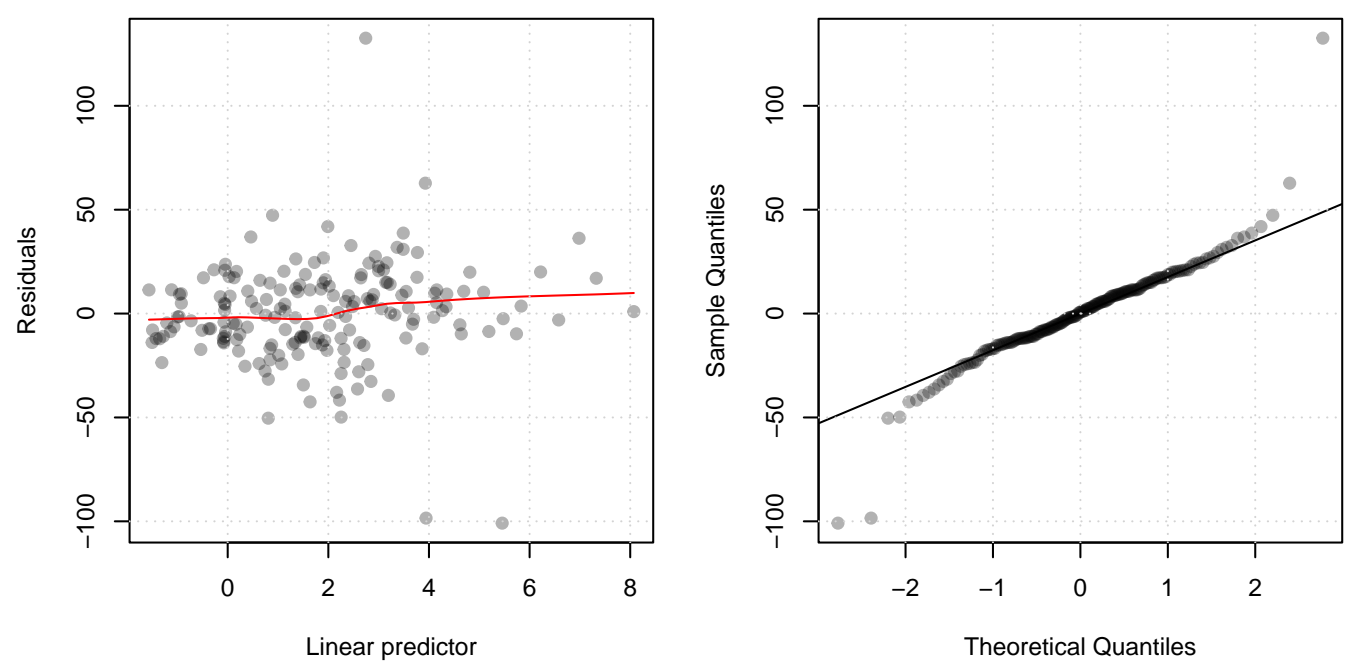

Figure 3: Residual plots of the normal linear mixed-effects transformation model fitted to the sleepstudy data. Left: Residuals plotted against the linear predictor. Right: QQ plot of the residuals against Gaussian quantiles.

'log Lik.' -201 (df=6)

The value of the log-likelihood is different because we are now calculating log-probabilities instead of log-densities of a continuous distribution. However, despite the decreased precision of the measurements, the parameter estimates are similar to what we got with the exactly observed outcomes.

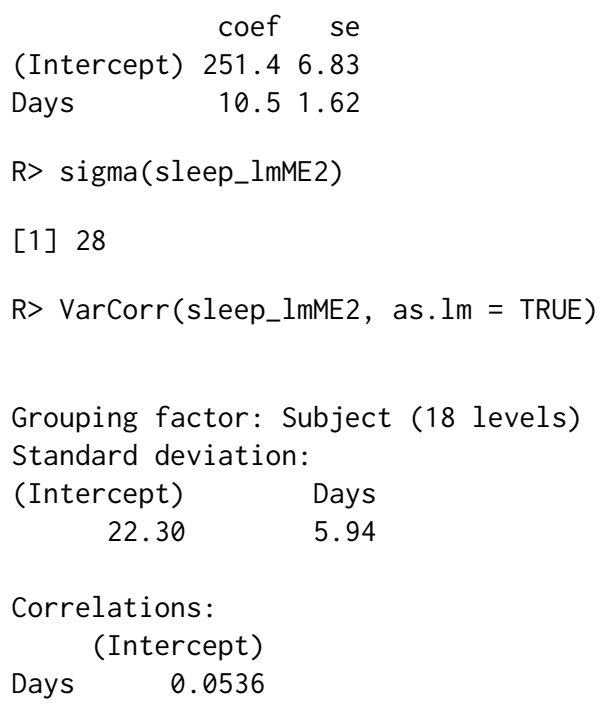

The small estimated value of the correlation coefficient between the random slope and intercept suggests that a model with independent random effects might be more appropriate. To estimate such a model, we can use the same notation as in lme4

R> sleep_lmME3 <- LmME(Reaction_ic Days + (Days || Subject), data = sleepstudy) R> logLik(sleep_lmME3)

'log Lik.' -201 (df=5)

Comparing the two models using a likelihood ratio test, we see no evidence against the more parsimonious model (sleep_lmME3).

R> anova(sleep_lmME2, sleep_lmME3) 
Model comparison

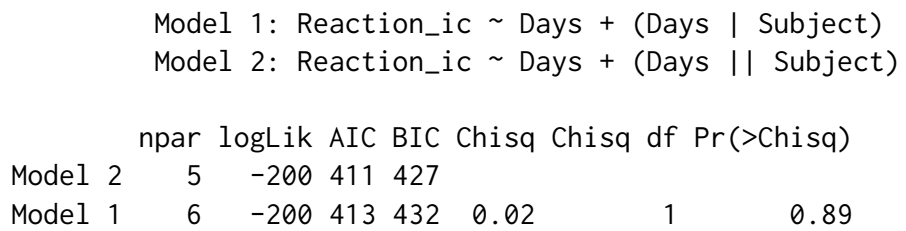

Note that the standard likelihood ratio tests provided by anova are very conservative for model comparisons that involve setting some of the random effects variances to zero due to the non-standard asymptotics of tests on the boundary of the parameter space (Self and Liang, 1987).

\section{Box-Cox-type mixed-effects models}

Substituting the linear baseline transformation function, $h(y)=\vartheta_{1}+\vartheta_{2} y$, with a general, monotonic increasing smooth function, we can relax the conditional normality assumption of the model discussed in Section 2.3.1. The transformation model approach proposed by Hothorn et al. (2018) uses Bernstein bases to approximate this general increasing function in a fully parametric manner, i.e., $h(y)=$ $\mathbf{a}_{\mathrm{Bs}, K+1}(y)^{\top} \boldsymbol{\vartheta}$. The resulting model can be regarded as a version of the Box-Cox regression (Box and Cox, 1964), where the transformation of the response is estimated simultaneously with the model parameters. It should be pointed out that, although its approach is similar in spirit, tramME does not use the Box-Cox power transformation to approximate $h(y)$. For an implementation utilizing the original Box-Cox transform in the context of mixed-effects models, see the R package boxcoxmix by Almohaimeed and Einbeck (2020).

A more flexible version of the model described in (3) will take the form

$$
\begin{aligned}
\mathbb{P}\left(\text { Reaction } \leq y \mid \text { Days, } \alpha_{i}, \beta_{i}\right) & =\Phi\left(\mathbf{a}(y)^{\top} \boldsymbol{\vartheta}-\beta \text { Days }-\gamma_{1 i}-\gamma_{2 i} \text { Days }\right) \\
\left(\begin{array}{l}
\gamma_{1 i} \\
\gamma_{2 i}
\end{array}\right) & \sim \mathcal{N}_{2}\left\{\left(\begin{array}{l}
0 \\
0
\end{array}\right),\left(\begin{array}{cc}
\tau_{1}^{2} & \tau_{12} \\
\tau_{12} & \tau_{2}^{2}
\end{array}\right)\right\} .
\end{aligned}
$$

The Box-Cox-type transformation mixed model can be estimated using the BoxCoxME() function of the tramME package. For this specific application, we set the order of the polynomials in Bernstein form to 10 .

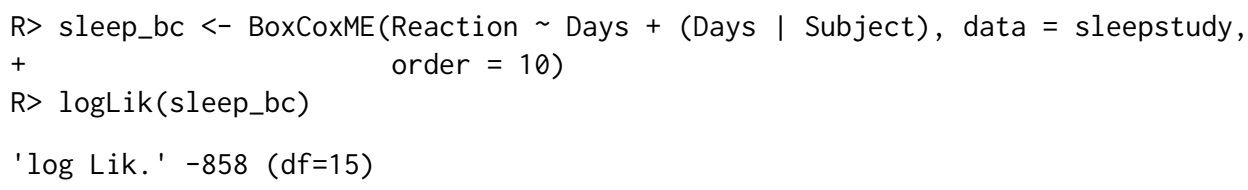

Note that the log-likelihood of this model is higher than that of the normal linear model because we are now approximating the baseline transformation function flexibly at the expense of a larger number of parameters.

The conditional quantiles calculated - using the same set of function calls, and with the same caveats, as in the analogous case of LmME - from the model defined by Equation (5) are shown in Figure 4. Comparing these results to Figure 2 reveals departures from conditional normality in the response distributions: At different lengths of sleep deprivation, the conditional distribution of the participants' reaction times is not a shifted normal distribution anymore, but it also changes its spread and shape.

The conditional distributions of the outcome can be further inspected visually with the plot method of tramME, which is designed to plot these distributions on a scale specified by the user. The left-hand side plot in Figure 5 compares the conditional densities of subjects 308 and 309 at various sleep deprivation lengths. Clearly, subject 308 is hardly affected by sleep deprivation because the mean and variance of the distribution of reaction time for this subject increase only marginally with days of sleep deprivation. In contrast, subject 309 showed longer mean reaction times and an increased variability of reaction times with increasing duration of sleep deprivation. The variance effect is not detectable from a classical normal linear mixed model but can be observed after a data-driven response transformation to normality. In the right panel of Figure 5, the conditional distribution of a hypothetical reference subject with zero random effects values is depicted. It should be noted that the latter is, in general, not equal to the marginal distribution of the outcome, which can be calculated by integrating the conditional distributions over the vector of random effects. We will return to this question at the end of this section.

The plots in Figure 5 can be generated with the commands 


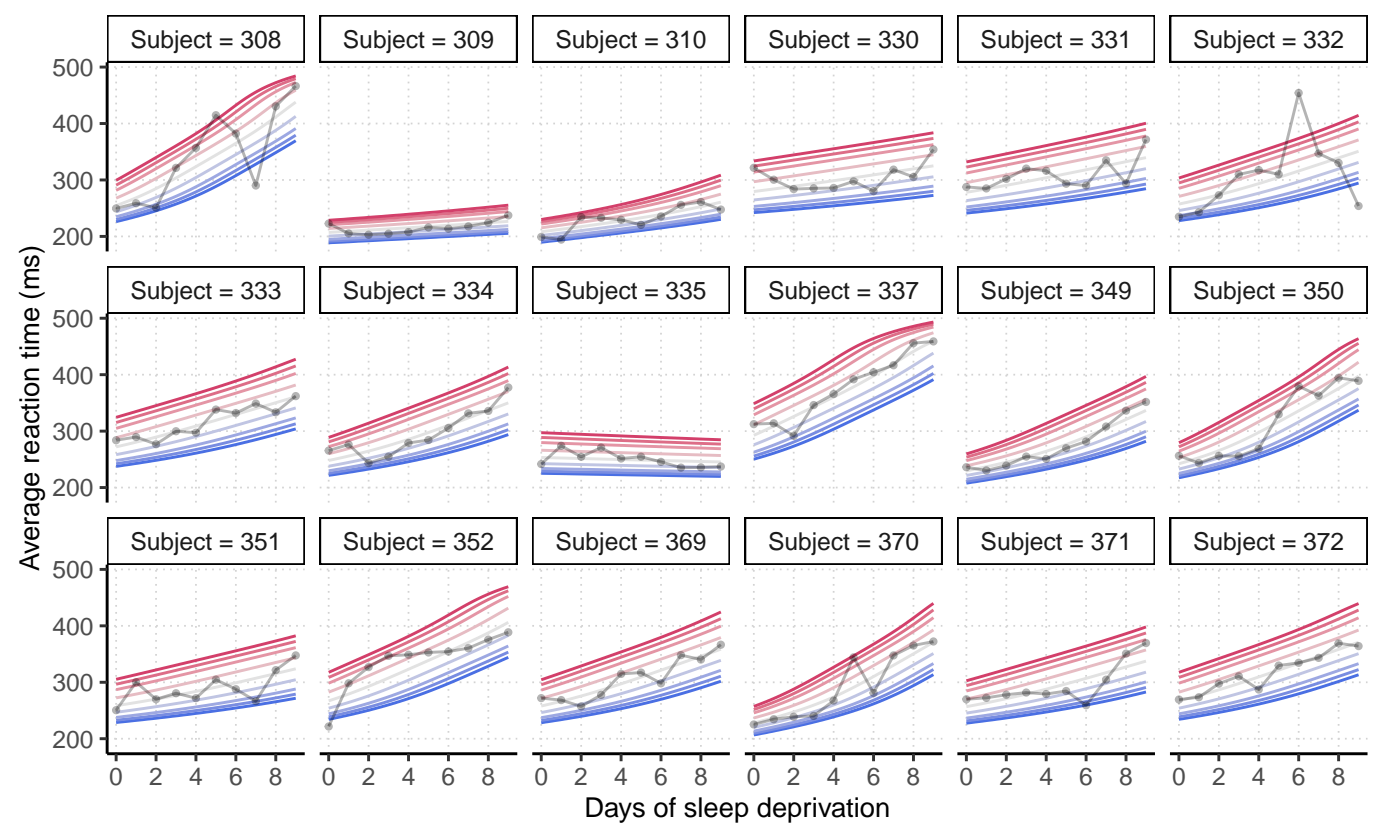

Estimated conditional quantiles

$$
-0.025-0.05-0.1-0.25-0.5-0.75-0.9-0.95-0.975
$$

Figure 4: Quantiles of the conditional distribution of the outcome fitted to the sleepstudy data with the non-normal (Box-Cox-type) linear mixed-effects transformation model (BoxCoxME) defined in Equation (5).
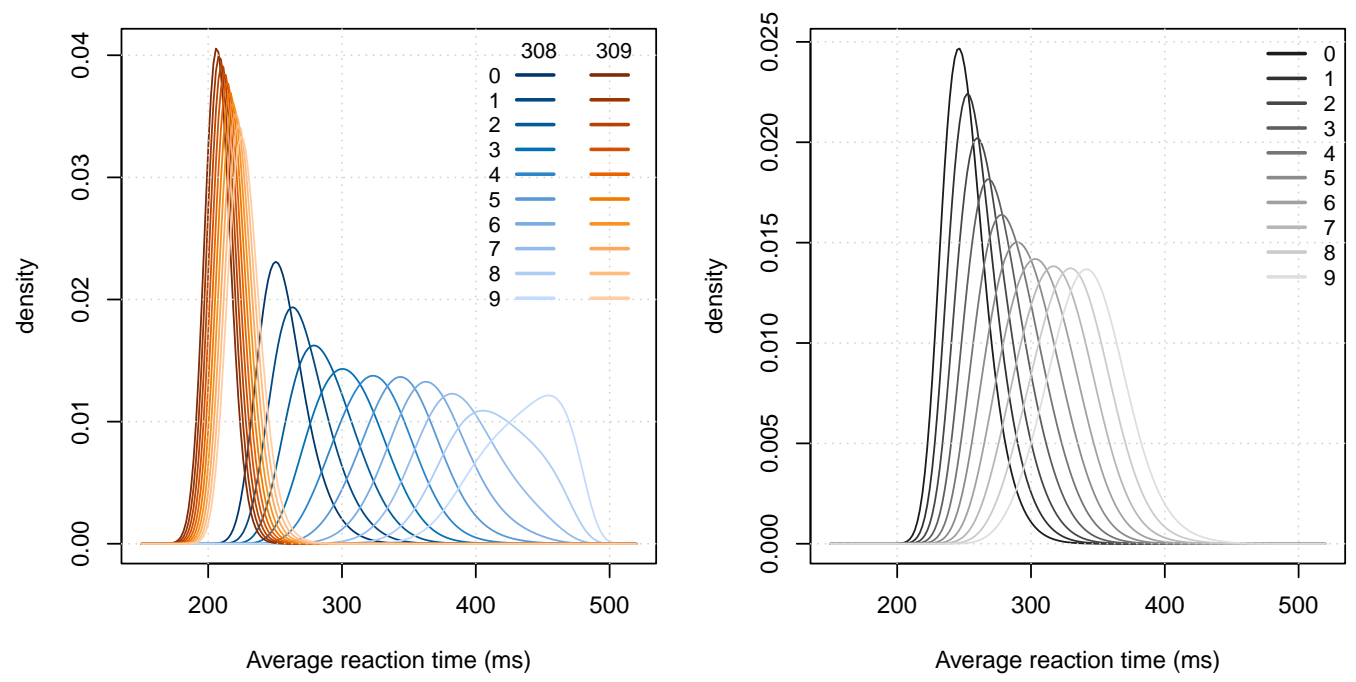

Figure 5: Conditional densities of the outcome from a non-normal (Box-Cox-type) mixed-effects linear transformation model (BoxCoxME) fitted to the sleepstudy data. Left: The conditional densities of subject 308 and 309 at various lengths of sleep deprivation (0-9 days). Right: The conditional densities of a reference subject (with random effects equal to zero) at various lengths of sleep deprivation (0-9 days). 
R> \#\# -- Compare two subjects (308 and 309)

$\mathrm{R}>\mathrm{nd}<-$ subset(sleepstudy, subset = Subject \%in\% c $(308,309)$ )

$\mathrm{R}>\operatorname{plot}($ sleep_bc1b, newdata $=$ nd, type $=$ "density", $\mathrm{K}=200$ )

R> \#\# -- The reference subject (at the mean of the random effect vector)

$\mathrm{R}>$ \#\# (we only need an arbitrary subject)

$\mathrm{R}>\mathrm{nd}<-$ subset( (leepstudy, subset $=$ Subject $==308$ )

$\mathrm{R}>$ \#\# NOTE: we explicitly set the random effects vector to 0

$\mathrm{R}>\operatorname{plot}($ sleep_bc1b, newdata $=$ nd, ranef $=$ "zero", type $=$ "density", $\mathrm{K}=200$ )

(and with some additional formatting steps that are omitted for the sake of brevity but can be found in the accompanying material).

In line with the methodology presented by Hothorn et al. (2018) and Hothorn (2020), we can define more complex mixed-effects transformation models by interacting the covariates with the basis expansion of the outcome. In the resulting extended model, the fixed effects are dependent on the level of the outcome. For the sleepstudy example, this model can be written as

$$
\begin{aligned}
\mathbb{P}\left(\text { Reaction } \leq y \mid \text { Days, } \alpha_{i}, \beta_{i}\right) & =\Phi\left(\mathbf{a}(y)^{\top} \boldsymbol{\vartheta}-\beta(y) \text { Days }-\gamma_{1 i}-\gamma_{2 i} \text { Days }\right) \\
\left(\begin{array}{l}
\gamma_{1 i} \\
\gamma_{2 i}
\end{array}\right) & \sim \mathcal{N}_{2}\left\{\left(\begin{array}{l}
0 \\
0
\end{array}\right),\left(\begin{array}{cc}
\tau_{1}^{2} & \tau_{12} \\
\tau_{12} & \tau_{2}^{2}
\end{array}\right)\right\},
\end{aligned}
$$

which is often referred to as "distribution regression" (Chernozhukov et al., 2013).

The model in Equation 6 can be defined in tramME using the I operator on the left-hand side of the model formula.

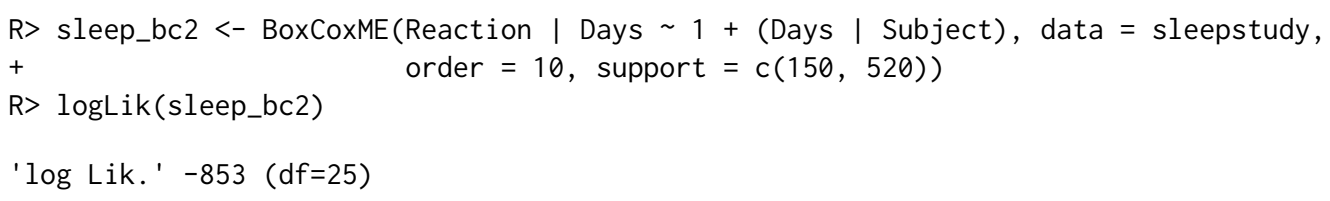

Plotting the conditional quantiles calculated from the resulting model in Figure 6 and comparing it with Figures 2 and 4 demonstrates the increased flexibility of the specification.

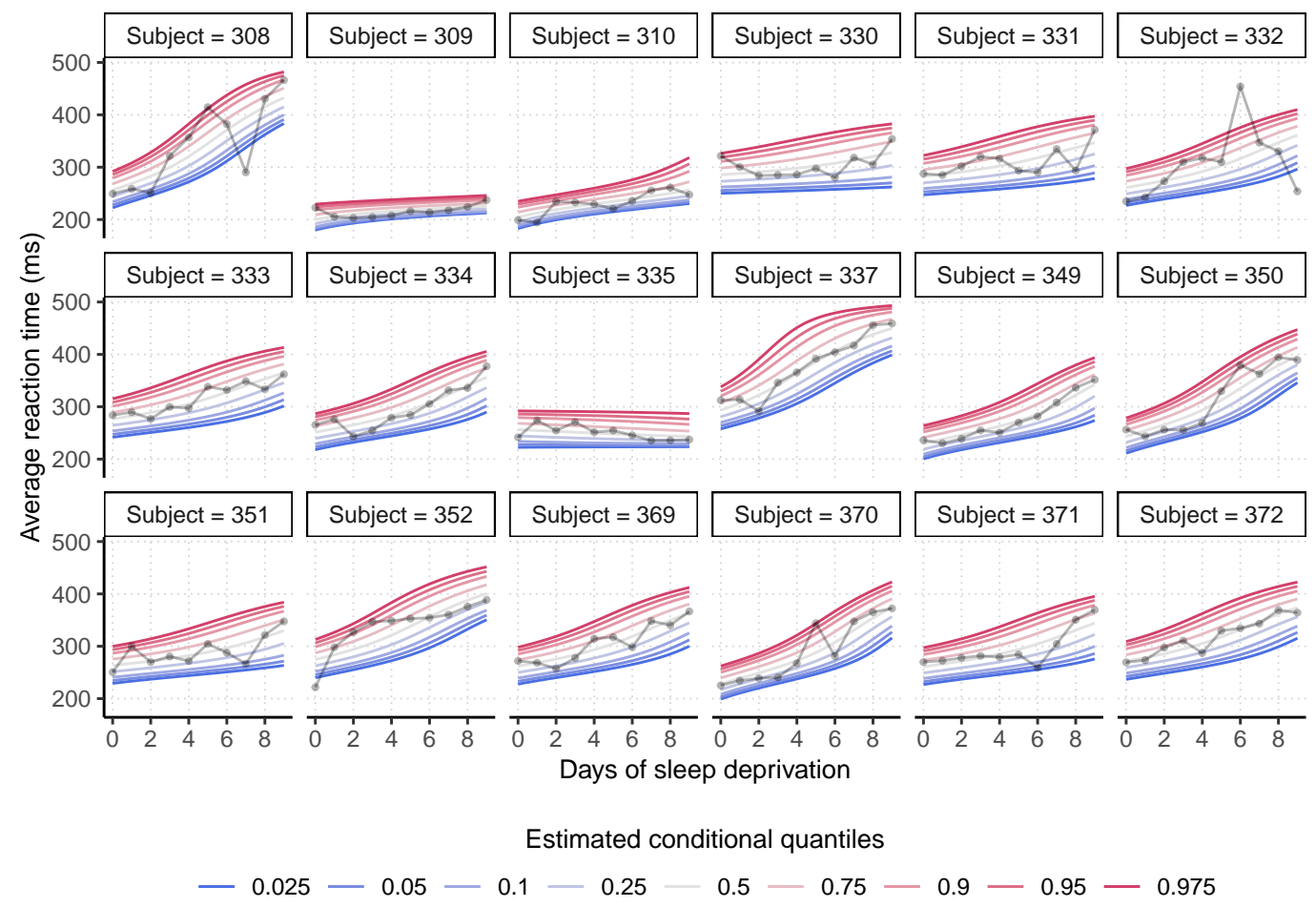

Figure 6: Quantiles of the conditional distribution of the outcome fitted to the sleepstudy data with the non-normal (Box-Cox-type) distributional mixed-effects transformation model (BoxCoxME) defined in Equation (6). 
In many cases, the goal of the analysis is to estimate the marginal distribution of the outcomes, i.e., integrating out the random effects from the conditional model (1). In the general formulation, there is no analytical solution for the integral, but we can use numerical methods to approximate the marginal distributions at various values of the outcome. The following code utilizes the simulate and predict methods implemented in the tramME package to get Monte Carlo estimates of the outcome distribution implied by the model (5).

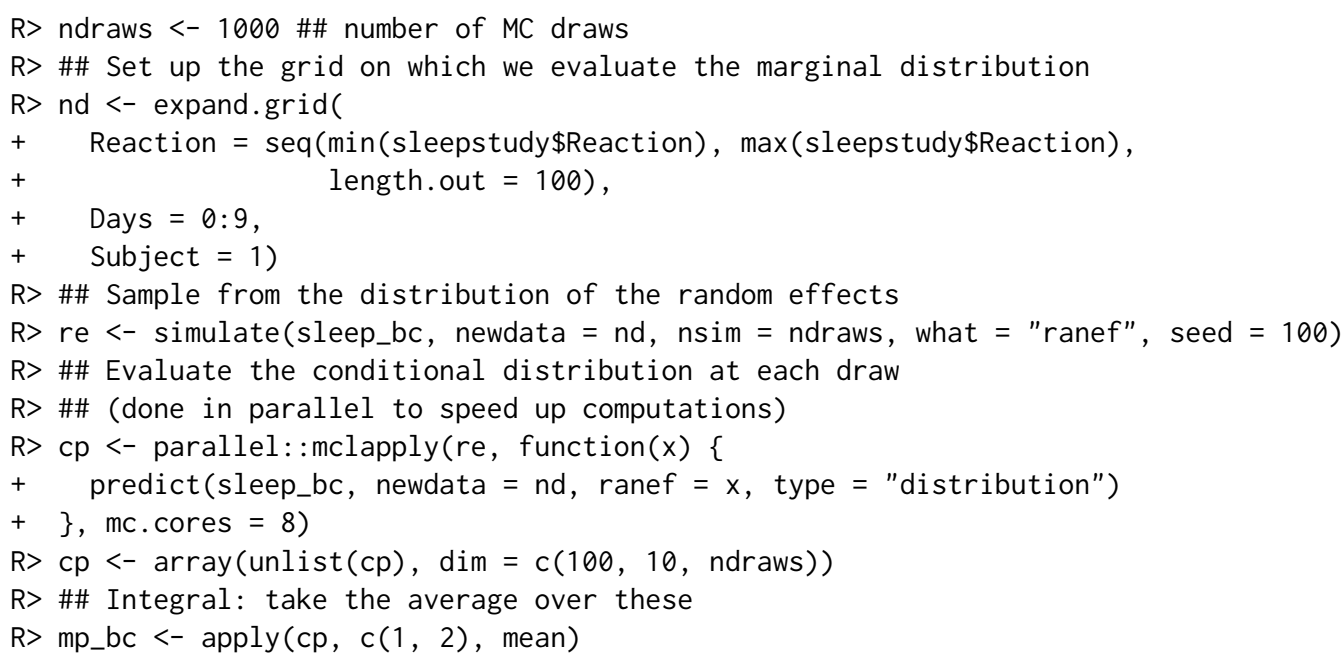

Figure 7 compares the conditional distributions obtained by integrating over the vector of random effects in models (3) and (5).
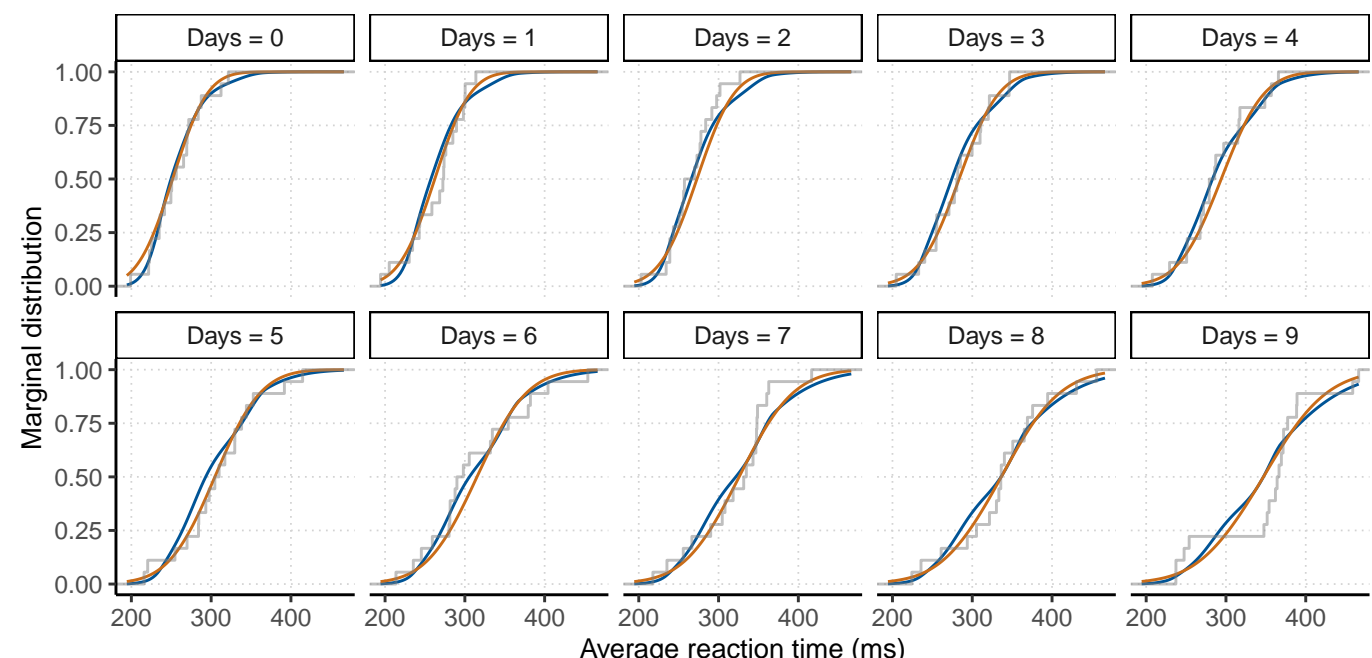

- BoxCoxME — LmME - ECDF

Figure 7: Comparison of the marginal distributions implied by the normal linear (LmME) and the Box-Cox-type (BoxCoxME) mixed-effects models fitted to the sleep deprivation study dataset. The empirical cumulative distribution functions (ECDF) are also plotted, conditionally on the days of sleep deprivation.

\section{Mixed-effects continuous outcome logistic regression}

The increased flexibility of the Box-Cox-type model, i.e., using a general baseline transformation function instead of a linear one, comes with the price that the coefficient estimates will not be easily interpretable as expected changes in the mean in the conditional model. Switching to the standard logistic error distribution provides a solution to this problem, as the parameter estimates in the resulting model can be interpreted as log-odds ratios. This continuous outcome logistic regression model was used by Lohse et al. (2017) to analyze body mass index (BMI) distributions. 
Manuguerra and Heller (2010) proposed a mixed-effects logistic regression model for bounded, continuous measurements of pain levels in a randomized, double-blind, placebo-controlled trial of low-level laser therapy for subjects with chronic neck pain presented by Chow et al. (2006). The levels of pain, measured on a visual analog scale, and normalized between 0 and 1, are plotted in Figure 8 for each subject at the different follow-up times.

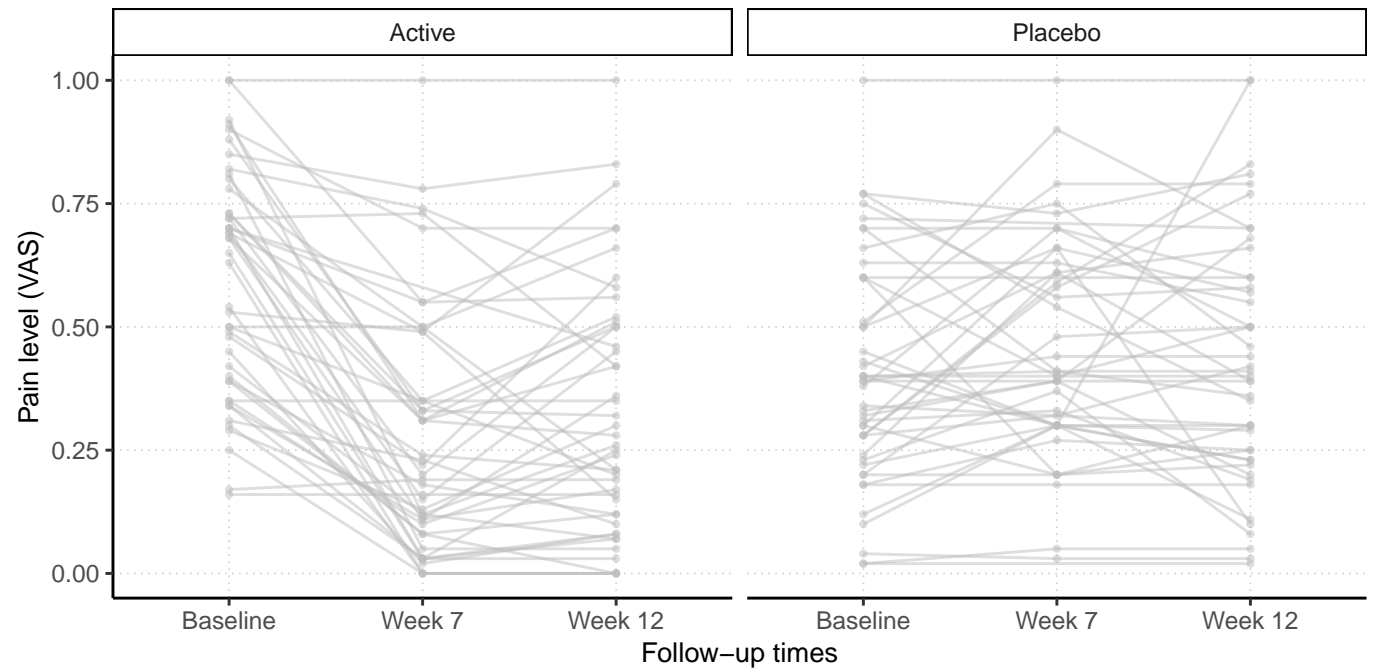

Figure 8: Neck pain dataset: Trajectories of pain levels measured on a visual analog scale (VAS) in the active treatment and placebo-controlled groups reported in Chow et al. (2006).

The mixed-effects model suggested by Manuguerra and Heller (2010) parameterizes the log-odds of experiencing smaller pain levels as a linear function of fixed and random effects and the baseline transformation. With the treatment group indicator, laser, and time denoting the follow-up times,

$$
\begin{aligned}
\log \left[\frac{\mathbb{P}\left(\text { pain } \leq y \mid \text { laser, time, } \alpha_{i}\right)}{\mathbb{P}\left(\text { pain }>y \mid \text { laser, time, } \alpha_{i}\right)}\right] & =h(y)+\beta_{\text {Active }}+\beta_{7 \mathrm{w}}+\beta_{12 \mathrm{w}}+\beta_{7 \mathrm{w}, \text { Active }}+\beta_{12 \mathrm{w}, \text { Active }}+\alpha_{i} \\
\alpha_{i} & \sim \mathcal{N}\left(0, \tau^{2}\right)
\end{aligned}
$$

where $h(y)$ is an increasing function of the outcome. Rearranging the terms in the model above reveals that this indeed is a mixed-effects transformation model, with the distribution function of the standard logistic distribution ("expit" function) as $F_{Z}$,

$$
\begin{gathered}
\mathbb{P}\left(\text { pain } \leq y \mid \text { laser, time, } \alpha_{i}\right)=\operatorname{expit}\left(h(y)+\beta_{\text {Active }}+\beta_{7 \mathrm{w}}+\beta_{12 \mathrm{w}}\right. \\
\left.+\beta_{7 \mathrm{w}, \text { Active }}+\beta_{12 \mathrm{w}, \text { Active }}+\alpha_{i}\right) \\
\alpha_{i} \sim \mathcal{N}\left(0, \tau^{2}\right) .
\end{gathered}
$$

The ColrME () function of the tramME package estimates mixed-effects continuous outcome logistic regression models using polynomials in Bernstein form to approximate $h(y)$. Applying this model to the neck_pain dataset:

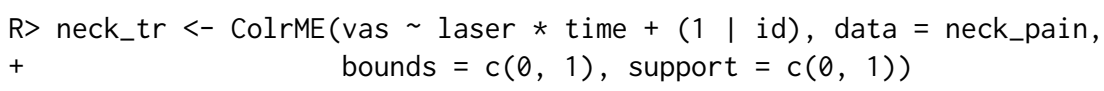

Notice that we explicitly set the bounds and the support of the outcome variable to $[0,1]$ because the pain levels are measured on a bounded scale.

The ordinalCont package by Manuguerra et al. (2020) implements an alternative formulation of the model (7) based on the method described in Manuguerra et al. (2017). In their approach, the baseline transformation is parameterized using B-splines and the estimation is carried out in a penalized likelihood framework.

R> library ("ordinalCont")

$\mathrm{R}>$ neck_ocm <- ocm(vas $\sim$ laser * time $+(1 \mid$ id $)$, data $=$ neck_pain, scale $=\mathrm{c}(\theta, 1))$

Figure 9 compares the results of the mixed-effects transformation model approach to the estimates obtained using the ordinalCont package. Because the two models are not exactly the same, we see 
some differences in the parameter estimates as well as in the fitted baseline transformation functions, but the two model fits are reasonably close to each other.
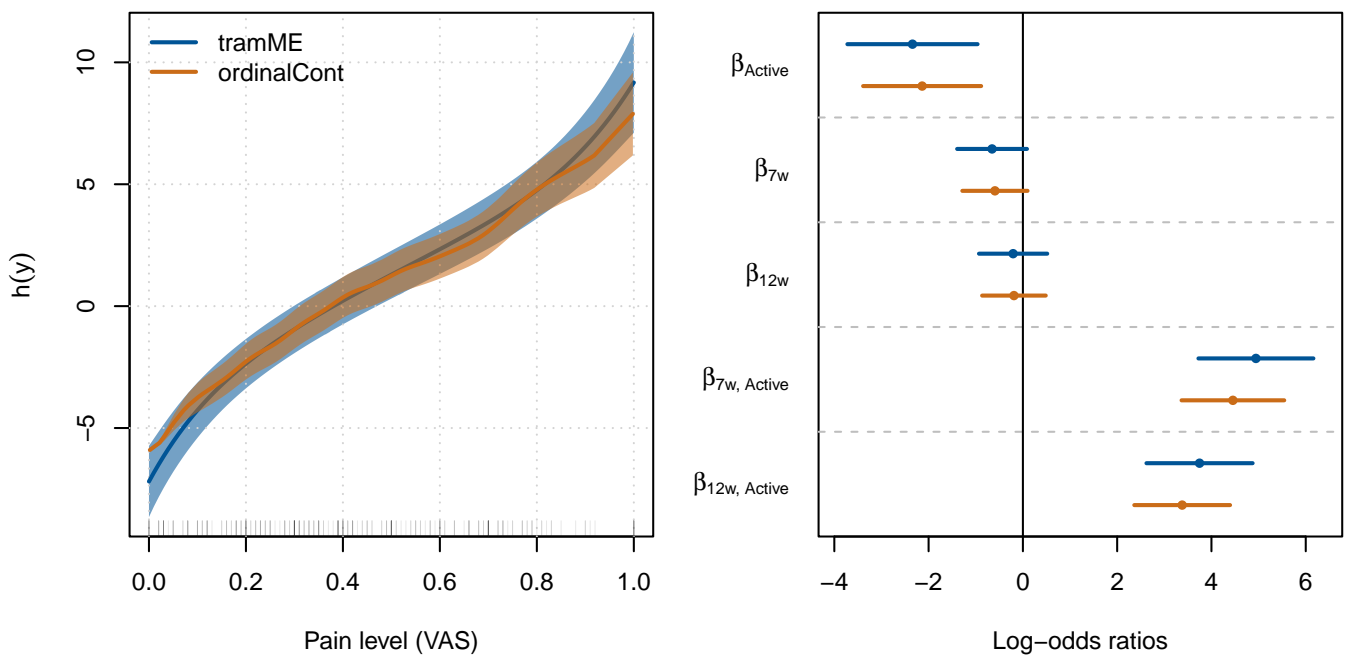

Figure 9: Left: Baseline transformations in continuous outcome logistic regressions estimated with the tramME and ordinalCont packages on the neck_pain dataset. The solid lines denote the point estimates, and the areas indicate the $95 \%$ point-wise confidence intervals. Right: Coefficient estimates from tramME and ordinalCont packages and their 95\% Wald confidence intervals.

The odds ratio estimates of the model fitted by $\operatorname{ColrME}()$,

$R>\exp ($ coef(neck_tr))
laser 1
time2
time3 laser1:time2 laser1:time3
0.0961
0.5200
0.8125
140.2076
42.4076

as well as the results from the ordinalCont package, suggest that there is an imbalance in the sample at baseline, i.e., the odds of experiencing less pain in the active treatment group is only about $10 \%$ that of in the control group for any pain levels. Based on the estimates, the treatment has a strong significant effect, especially at the seven-week follow-up, but seems to level off after 12 weeks.

If we want to compare the marginal distributions in the treatment and control groups directly, we have to average over the distribution of the random effects. Because we only have a random intercept in this example, we have to evaluate a one-dimensional integral. We could use the same Monte Carlo method as we did in Section 2.3.2, or we can apply the adaptive quadrature method implemented in the stats package of $\mathrm{R}$. The example below uses this approach to demonstrate the multiple options the user has in dealing with such problems.

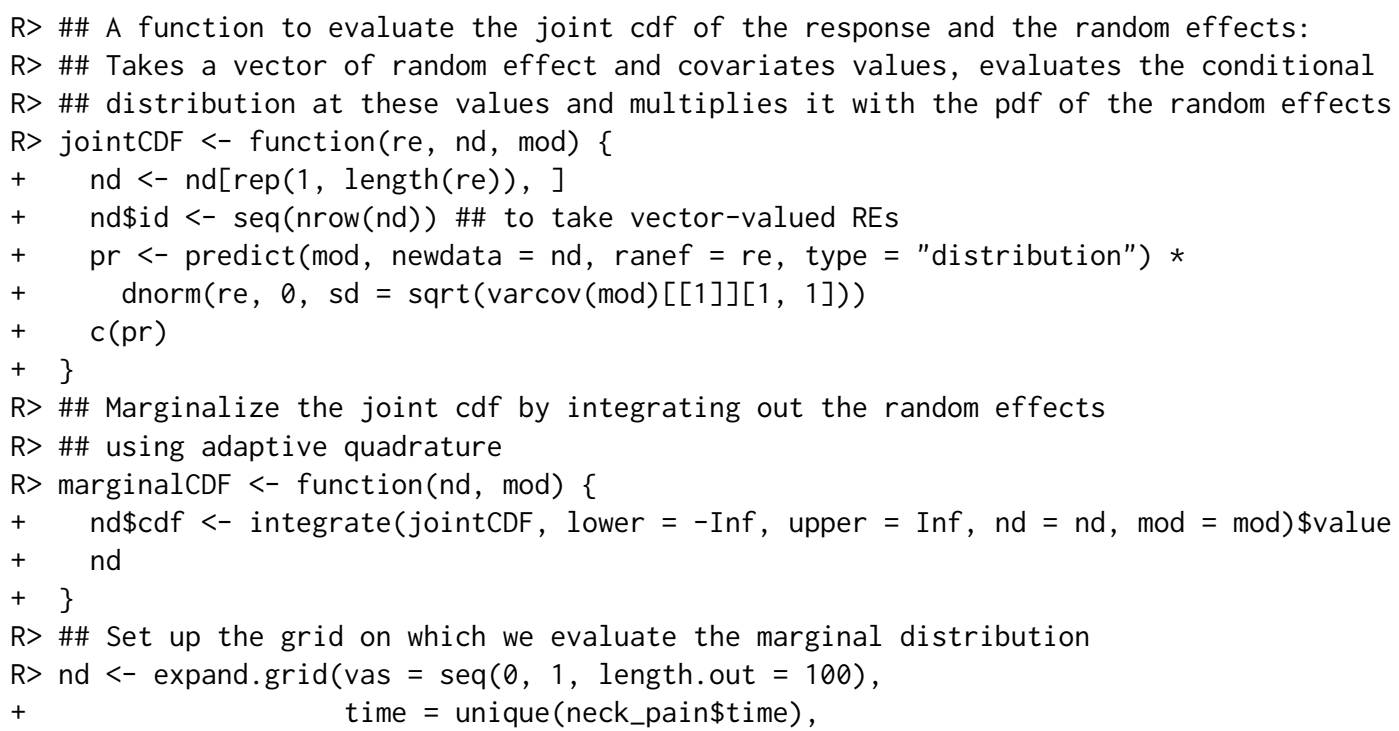


$\mathrm{R}>$ \#\# Calls marginalCDF on each row of nd

$\mathrm{R}>$ \#\# (done in parallel to speed up computations)

R> mp_colr <- parallel: :mclapply(split(nd, seq(nrow(nd))),

$+\quad$ marginalCDF, mod $=$ neck_tr, $\mathrm{mc}$. cores $=8$ )

R> mp_colr <- do.call("rbind", mp_colr)

Figure 10 compares the marginal distributions at different time points and confirms our previous conclusions on baseline imbalance and treatment effect dynamics.

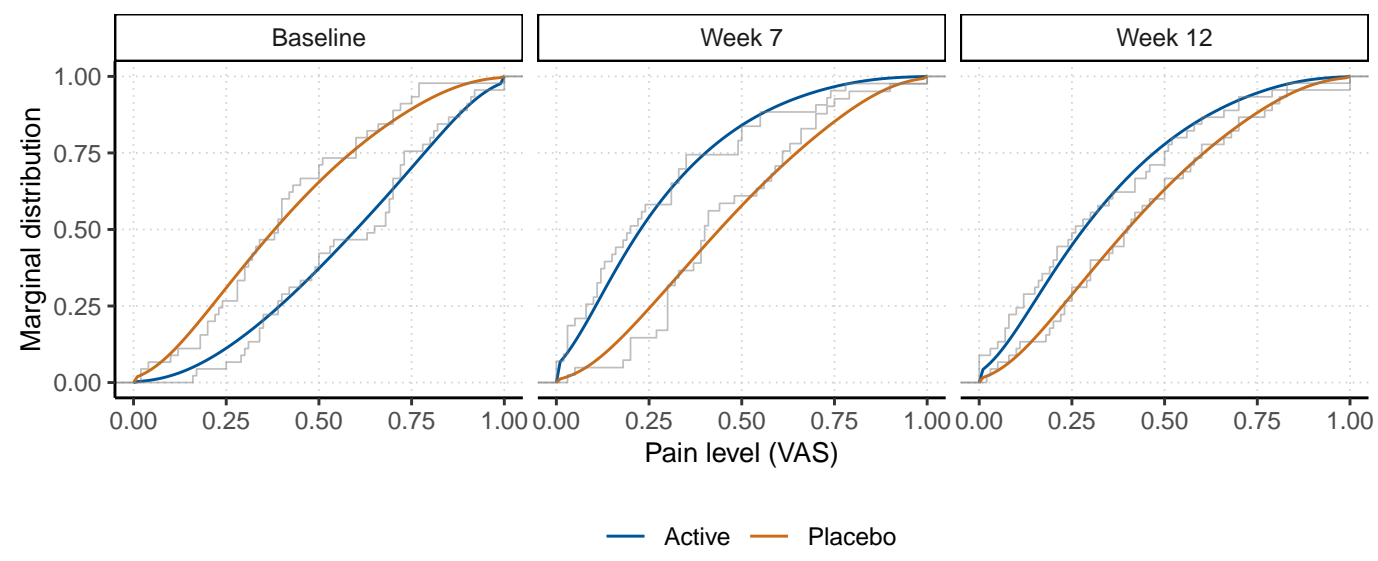

Figure 10: Comparison of marginal distributions, calculated from a mixed-effects continuous outcome logistic regression model in the treatment (Active) and control (Placebo) groups at baseline and the two follow-up times. The step functions represent the empirical cumulative distribution functions of the specific groups.

\section{Mixed-effects transformation models for time-to-event outcomes}

Mixed models for right-censored data are important in survival analysis and we consider the example dataset eortc in the coxme package by Therneau (2020). This simulated dataset emulates the structure of the outcomes of a breast cancer trial by the European Organization for Research and Treatment of Cancer, and consists of 2323, possibly right-censored, data points from 37 enrolling centers. We define a proportional hazards mixed-effects model with random center $(i=1, \ldots, 37)$ and treatment $(\operatorname{tr} t)$ effects (nested within centers and indexed by $j=0,1$ ).

$$
\begin{aligned}
\mathbb{P}\left(Y \leq y \mid \operatorname{trt}, \gamma_{1 i}, \gamma_{2 j(i)}\right) & =1-\exp \left(-\exp \left(h(y)+\beta_{\text {trt }}+\gamma_{1 i}+\gamma_{2 j(i)}\right)\right) \\
\gamma_{1 i} & \sim \mathcal{N}\left(0, \tau_{1}^{2}\right), \quad \gamma_{2 j(i)} \sim \mathcal{N}\left(0, \tau_{2}^{2}\right)
\end{aligned}
$$

This model corresponds to a mixed-effects transformation model with the minimum extreme value distribution as the error distribution. Treating the baseline transformation as a general smooth function, approximated using polynomials in Bernstein form, we get the fully parametric version of the Cox proportional hazards model with normally distributed random effects.

We can fit this model with the CoxphME() function of tramME.

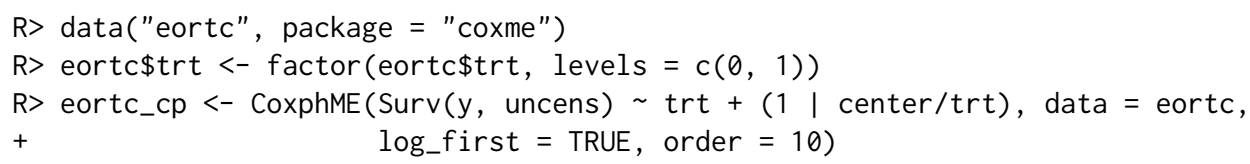

The nested random effects structure is defined with the / operator. The log_first = TRUE option casts the outcome variable to the log-scale before defining the Bernstein bases, which usually improves the model fit when dealing with skewed conditional distributions, while we explicitly set the order of the polynomials in Bernstein form with order $=10$. The confidence interval for the treatment effect (transformed to the hazard ratio scale) suggests evidence for the effectiveness of the treatment,

$\mathrm{R}>\exp ($ confint (eortc_cp, parm $=$ "trt1", estimate $=$ TRUE) $)$ 
lwr upr est

trt1 $1.8 \quad 2.512 .12$

while the profile intervals of the random effects standard deviations indicate similar magnitude of center-level and treatment-level (within center) variabilities.

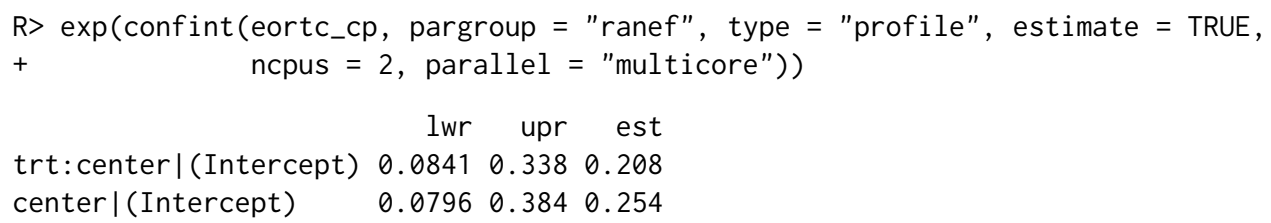

The transformation model framework by Hothorn (2020) allows for stratification, i.e., specifying separate transformation functions for different groups defined by a stratification factor. Timedependent effects for the covariates can be introduced in the same way as in distribution regression to relax the proportionality assumption of the Cox model. To check the appropriateness of the proportional hazards assumption between treatment and control groups visually, we re-estimate the model stratifying for the treatment indicator, i.e., fitting transformation functions for the treatment and control groups separately, and inspect whether these two functions, which are the log-cumulative hazards when the error distribution is the minimum extreme value distribution, are parallel.

$\mathrm{R}>$ eortc_cp2 <- $\operatorname{CoxphME}(\operatorname{Surv}(\mathrm{y}$, uncens $) \mid 0+\operatorname{trt} \sim \theta+(1 \mid$ center/trt $)$, data $=$ eortc, $+\quad$ log_first $=$ TRUE, order $=10$ )

$\mathrm{R}>\operatorname{tr}<-$ trafo(eortc_cp2, confidence = "interval")

Figure 11 plots the stratified transformation functions against log-time. The two curves are very close to parallel, which indicates that the treatment effect is constant over time, i.e., the proportionality assumption is appropriate in the original model specification.

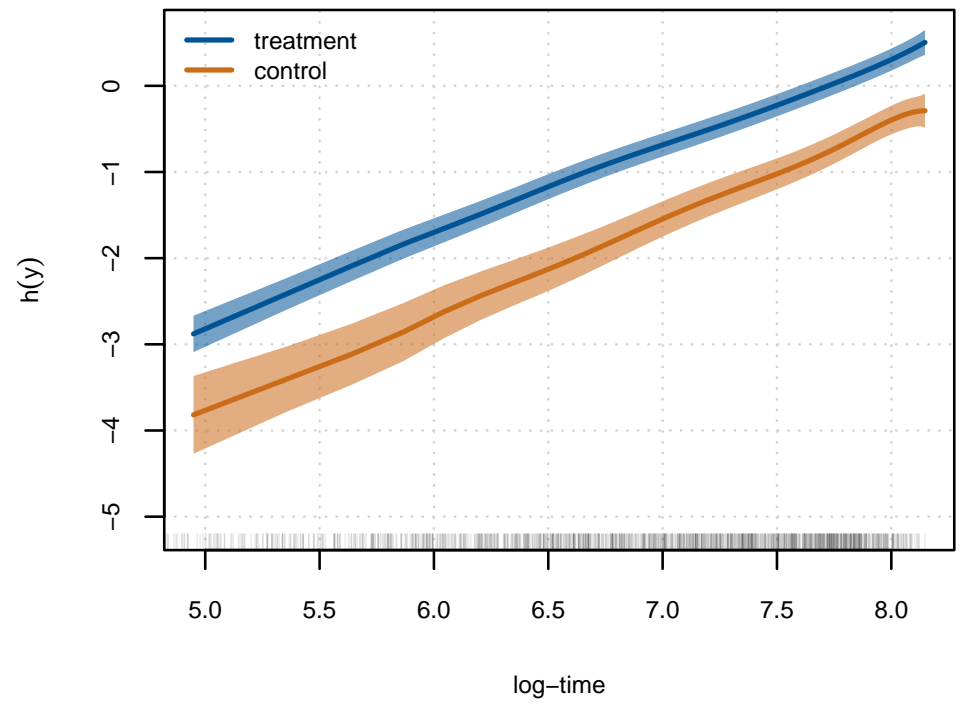

Figure 11: Comparison of baseline transformation functions in treatment and control groups, estimated using a stratified parametric mixed-effects Cox proportional hazards model on the eortc dataset.

In addition to proportionality, Figure 11 reveals another important aspect of the data generating process. The fact that the baseline log-cumulative hazards are linear in log-time suggests that the conditional distributions are close to the Weibull distribution, i.e., we can substitute the general baseline transformation function with $h(y)=\vartheta_{1}+\vartheta_{2} \log (y)$. Flipping the signs of the fixed and random effects terms of (8) and substituting the log-linear function to the baseline transformation, we get the model

$$
\begin{aligned}
\mathbb{P}\left(Y \leq y \mid \operatorname{trt}, \gamma_{1 i}, \gamma_{2 j(i)}\right) & =1-\exp \left(-\exp \left(\vartheta_{1}+\vartheta_{2} \log (y)-\beta_{\operatorname{trt}}-\gamma_{1 i}-\gamma_{2 j(i)}\right)\right) \\
\gamma_{1 i} & \sim \mathcal{N}\left(0, \tau_{1}^{2}\right), \quad \gamma_{2 j(i)} \sim \mathcal{N}\left(0, \tau_{2}^{2}\right) .
\end{aligned}
$$

The SurvregME() function of the tramME package implements a variety of parametric mixedeffects models that represent specific choices of the error distribution and the baseline transformation 
function in the general formulation of Equation (1). There are several other R packages available for estimating parametric survival models with mixed effects, such as parfm by Munda et al. (2012) and frailtypack by Rondeau et al. (2012). However, they typically do not allow for nested random-effects structures when assuming (log-)normally distributed frailty terms.

Fitting a mixed-effects Weibull model to the eortc dataset:

$\mathrm{R}>$ eortc_w <- SurvregME(Surv(y, uncens $) \sim \operatorname{trt}+(1 \mid$ center/trt $)$, data = eortc,

$+\quad$ dist $=$ "weibull")

Comparing the parameter estimates of the Cox proportional hazards model to those from the mixed-effects Weibull model,

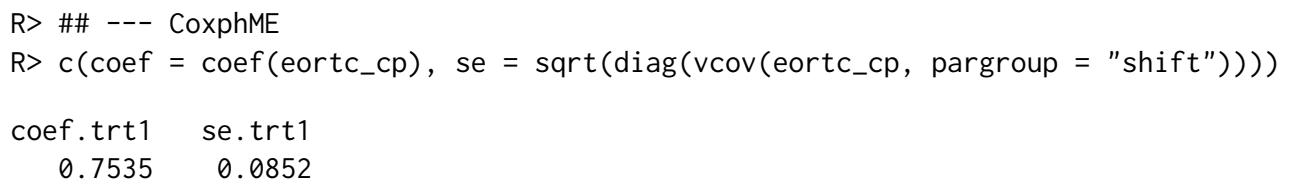

$\mathrm{R}>\operatorname{VarCorr}($ eortc_cp)

Grouping factor: trt:center (74 levels)

Standard deviation:

(Intercept)

0.208

Grouping factor: center (37 levels)

Standard deviation:

(Intercept)

0.254

$\mathrm{R}>$ \#\# --- SurvregME

$R>c(\operatorname{coef}=-\operatorname{coef}($ eortc_w $)$, se $=\operatorname{sqrt}(\operatorname{diag}(\operatorname{vcov}($ eortc_w, pargroup $=$ "shift") $)))$

coef.trt1 se.trt1

$0.7531 \quad 0.0851$

$\mathrm{R}>\operatorname{VarCorr}($ eortc_w)

Grouping factor: trt:center (74 levels)

Standard deviation:

(Intercept)

0.208

Grouping factor: center (37 levels)

Standard deviation:

(Intercept)

0.255

as well as their log-likelihood values

$R>c(\log L i k($ eortc_cp), $\operatorname{logLik}($ eortc_w $))$

[1] $-13027-13032$

confirms our suspicion that the dataset was indeed simulated from a conditional Weibull model.

Finally, we can compare the results from tramME to parameters estimated with the $\mathrm{R}$ package coxme.

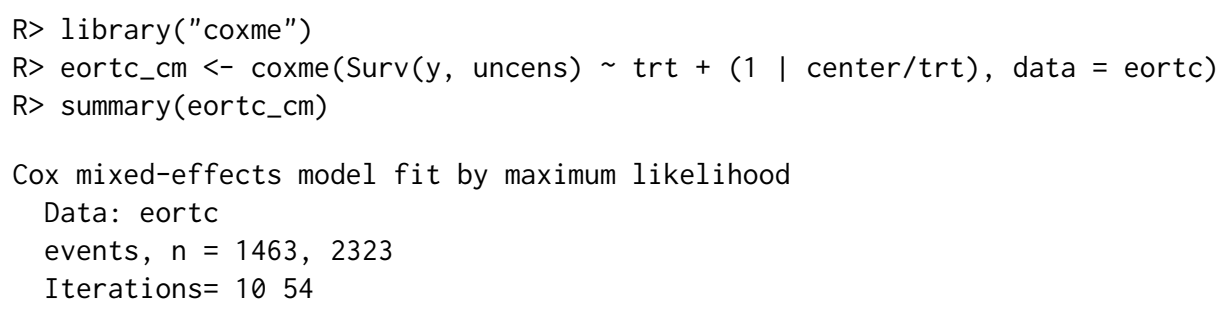


NULL Integrated Fitted

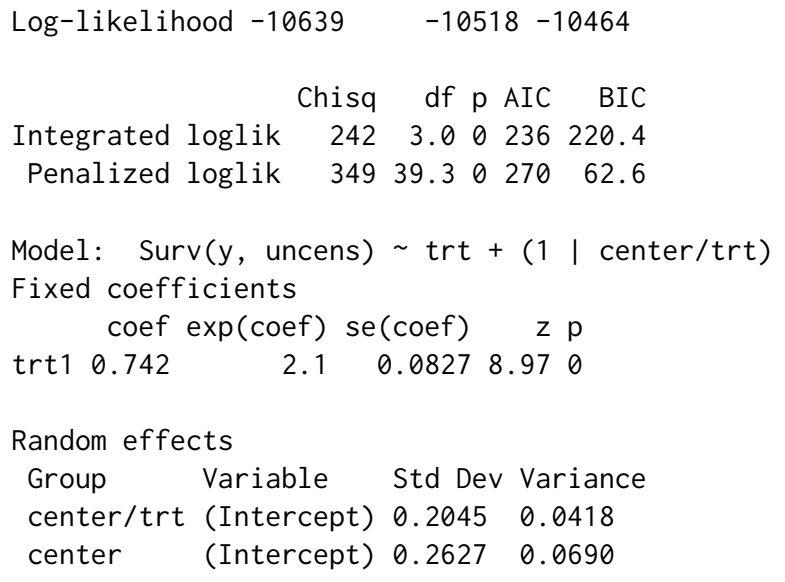

This package follows a different approach to estimate a mixed-effects Cox model by leaving the baseline hazards unspecified and maximizing the integrated partial likelihood. As a result, the parameter estimates are slightly different from the ones we got using the CoxphME() function, but the results are comparable, and the conclusions are identical, nevertheless.

\section{Mixed-effects transformation models for discrete ordinal outcomes}

Our last example demonstrates how the mixed-effects transformation framework can be used in modeling correlated discrete ordinal outcomes. As an example, we take the soup tasting dataset by Christensen et al. (2011). The dataset contains 1847 observations from 185 respondents in a soup tasting experiment. The subjects were familiarized with a reference product prior to the experiment and, during the experiment, were asked to distinguish between samples from the reference product and test product using a six-level ordinal scale indicating their level of confidence. The scale ranges from "reference, sure" (sureness $=1$ ) to "not reference, sure" (sureness $=6$ ). Figure 12 presents the proportions of response categories for the test and reference samples for respondent groups defined by how often they consume soup.
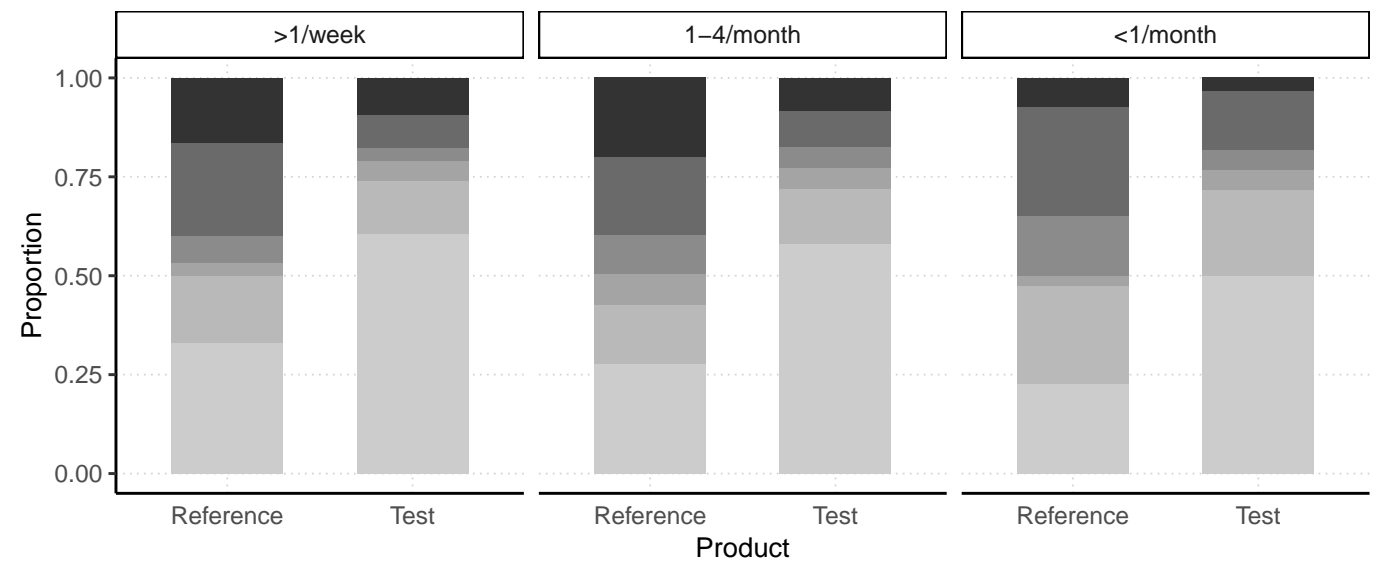

Sureness
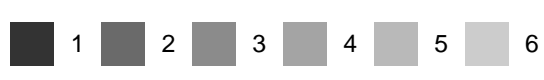

Figure 12: Data from the soup tasting study reported by Christensen et al. (2011): Proportions of responses of sureness levels (six levels, ranging from "reference, sure" to "not reference, sure") after tasting test and reference products. The data points are grouped by how often the respondents consume soup (more than once a week, one to four times a month, less than once a month).

Let us assume that we are interested in comparing the distributions of sureness ratings for reference products and test products while taking the repeated nature of the design into account. Moreover, in doing so, we also want to control for how often the respondents usually consume soup (denoted by the covariate freq). With $k=1 \ldots 5$, indicating the sureness levels except the last one, $i=1 \ldots, 185$ indexing the respondents, and $j=0,1$ indexing the reference and test products (covariate prod), 
respectively, the regression model we estimate can be written as

$$
\begin{gathered}
\mathbb{P}\left(\text { sureness } \leq k \mid \text { prod, freq, } \gamma_{1 i}, \gamma_{2 j(i)}\right)=\Phi\left(\vartheta_{k}-\beta_{\text {test }}-\beta_{1-4 / \text { month }}-\beta_{<1 / \text { month }}\right. \\
\left.-\gamma_{1 i}-\gamma_{2 j(i)}\right) \\
\gamma_{1 i} \sim \mathcal{N}\left(0, \tau_{1}^{2}\right), \quad \gamma_{2 j(i)} \sim \mathcal{N}\left(0, \tau_{2}^{2}\right) .
\end{gathered}
$$

The PolrME() function of the tramME package estimates models for ordered discrete outcomes. Depending on the choice of the error distribution, the user can fit proportional odds (logistic distribution), ordinal probit (standard normal distribution), proportional hazards (minimum extreme value distribution), or cumulative maximum extreme value models. In our example, we set method = 'probit' to estimate the probit model,

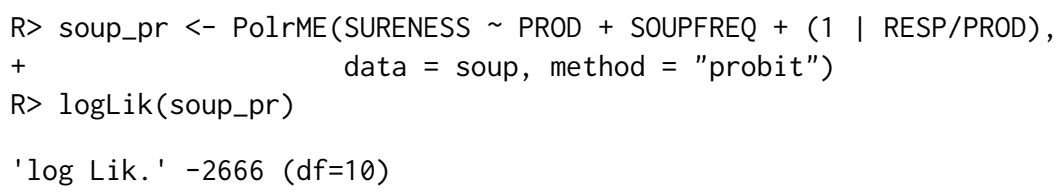

The R package ordinal by Christensen (2019) also implements mixed-effects regression models for ordered discrete outcomes. As a cross-check, we can re-estimate the same model with the function $\operatorname{clmm}()$,

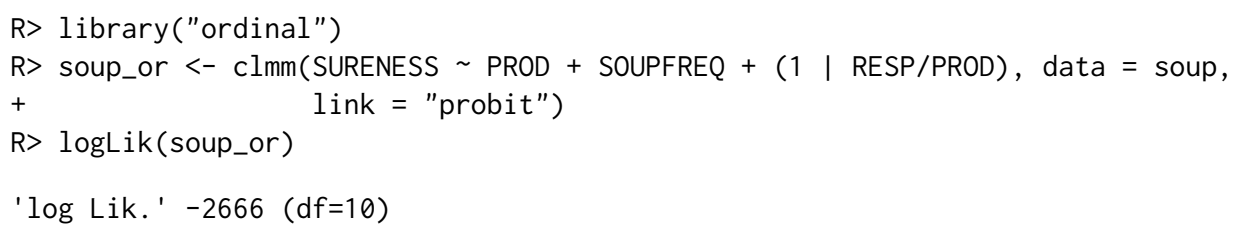

Based on the likelihood values and the parameter estimates,

R> $\max ($ abs (coef(soup_or) $-\operatorname{coef}($ soup_pr, with_baseline = TRUE) $)$ )

[1] $1.76 \mathrm{e}-05$

the results are essentially the same.

We can introduce non-proportional effects in the transformation model framework by stratifying on a covariate. In our example, we might want to extend the model to allow for different effect sizes of the soup consumption frequency covariate, depending on the level of the outcome variable. Rewriting model (9),

$$
\begin{aligned}
\mathbb{P}\left(\text { sureness } \leq k \mid \text { prod, freq, } \gamma_{1 i}, \gamma_{2 j(i)}\right) & =\Phi\left(\vartheta_{k}-\beta_{\text {test }}-\beta_{1-4 / \text { month }, k}-\beta_{<1 / \text { month }, k}-\gamma_{1 i}-\gamma_{2 j(i)}\right) \\
\gamma_{1 i} & \sim \mathcal{N}\left(0, \tau_{1}^{2}\right), \quad \gamma_{2 j(i)} \sim \mathcal{N}\left(0, \tau_{2}^{2}\right),
\end{aligned}
$$

and estimating it with tramME by stratifying for the soup frequency factor

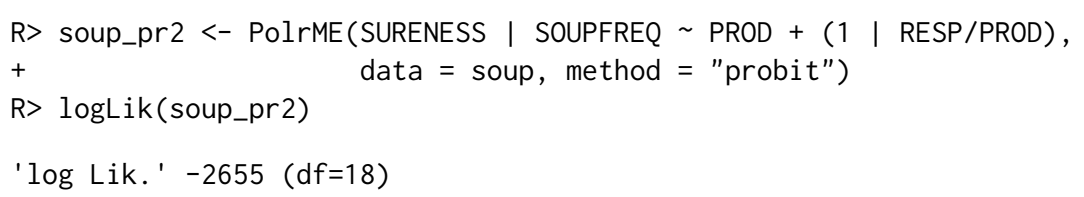

The likelihood ratio test comparing the two specifications suggests some evidence that the extended, partially proportional model fits the data better.

R> anova(soup_pr, soup_pr2)

Model comparison

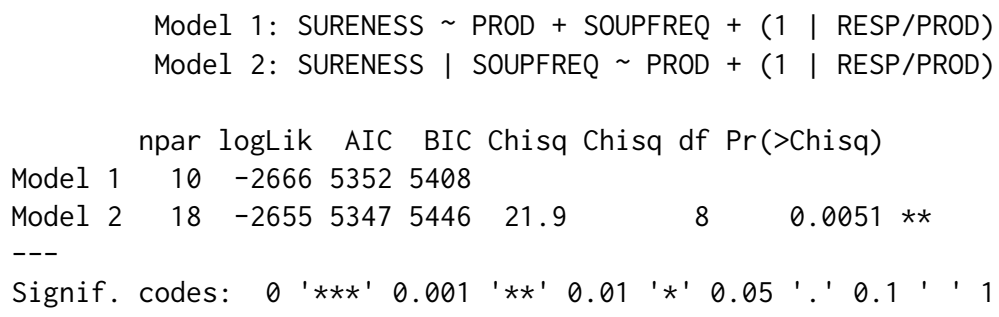




\section{Discussion}

Building the implementation of mixed-effects transformation models on the package TMB leads to significant efficiency gains in the computationally intensive steps of the maximum likelihood estimation. This computational efficiency is partly due to the use of Laplace approximation to integrate over the vector of random effects. However, several sources point out that Laplace's method can lead to biased estimates in some distributional settings. Pinheiro and Chao (2006) provide detailed numerical comparisons of the Laplacian approximation to adaptive Gaussian quadrature algorithms in the context of multilevel generalized models. Joe (2008) evaluates the method in the case of discrete outcome mixed-effects models and concludes that the inaccuracy increases with the amount of discreteness of the response variable and decreases as the cluster sizes increase.

It is worth mentioning that the conditional approach of modeling the distribution of the response, which is the basis of the transformation models implemented in the tramME package, is not the only way one could approach the problem of correlated outcomes in regression settings. The main alternative to a conditional (mixed-effects) modeling approach is a marginal model that parameterizes the marginal distribution of the outcome and treats the covariance structure as nuisance parameters. Generalized estimating equations (GEE, Hardin and Hilbe, 2013) models represent prominent examples of such an approach. Proponents of marginal models point out that, in a conditional model, the fixed effects parameter estimates cannot be interpreted as population averages, which is usually of primary interest in a regression analysis. Lindsey and Lambert (1998) emphasize that marginal parameter estimates from longitudinal studies can only be interpreted as population averages when the participants are representative to their populations, which is usually not the case. Moreover, they argue that defining models based on marginal distributions very often leads to complicated and implausible conditional distributions, whereas conditional models can more easily express physiologically plausible mechanisms on the level of the individual. Lee and Nelder (2004) argue that conditional models are more fundamental as they allow for both marginal and conditional inferences, which is not true in the case of marginal models. As we demonstrated in Sections 2.3.2 and 2.3.3, the marginal distributions implied by the conditional transformation model can be easily approximated using numerical techniques.

The tramME package, introduced in this article, extends the available options for modeling grouped data structures with mixed-effects regressions in several ways: Through its dense code base, tramME provides a unified and efficient estimation framework for a broad range of regression models. Examples in Section 2.3 demonstrate that using this single package, several very specific regression problems can be addressed. Relying only on a limited number of packages, in turn, decreases the likelihood of errors in the statistical analysis. As the examples show, the modular structure of our approach naturally leads to extensions of existing models (such as accounting for censoring or introducing nested or crossed random effects structures) that would otherwise require a lot of effort to re-implement from scratch. Moreover, the underlying theory of linear transformation models provides a flexible basis for the implementation of the package and for its future extensions.

\section{Acknowledgments}

This work was supported by the Swiss National Science Foundation, grant number 200021_184603.

\section{Bibliography}

A. Almohaimeed and J. Einbeck. boxcoxmix: Box-Cox-Type Transformations for Linear and Logistic Models with Random Effects, 2020. URL https://CRAN.R-project.org/package=boxcoxmix. R package version 0.28. [p405]

D. Bates, M. Mächler, B. M. Bolker, and S. Walker. Fitting Linear Mixed-Effects Models Using lme4. Journal of Statistical Software, 67(1):1-48, 2015. URL https://doi.org/10.18637/jss.v067.i01. [p398]

G. Belenky, N. J. Wesensten, D. R. Thorne, M. L. Thomas, H. C. Sing, D. P. Redmond, M. B. Russo, and T. J. Balkin. Patterns of performance degradation and restoration during sleep restriction and subsequent recovery: a sleep dose-response study. Journal of Sleep Research, 12(1):1-12, 2003. URL https://doi.org/10.1046/j.1365-2869.2003.00337.x. [p400,401]

G. E. P. Box and D. R. Cox. An analysis of transformations. Journal of the Royal Statistical Society. Series B (Methodological), 26(2):211-252, 1964. URL https://doi .org/10.1111/j.2517-6161.1964. tb00553. x. [p405] 
M. E. Brooks, K. Kristensen, K. J. van Benthem, A. Magnusson, C. W. Berg, A. Nielsen, H. J. Skaug, M. Mächler, and B. M. Bolker. glmmTMB balances speed and flexibility among packages for zero-inflated generalized linear mixed modeling. The R Journal, 9(2):378-400, 2017. URL https: //doi.org/10.32614/RJ-2017-066. [p400]

V. Chernozhukov, I. Fernández-Val, and B. Melly. Inference on counterfactual distributions. Econometrica, 81(6):2205-2268, 2013. URL https://doi .org/10.3982/ECTA10582. [p407]

R. T. Chow, G. Z. Heller, and L. Barnsley. The effect of $300 \mathrm{~mW}, 830 \mathrm{~nm}$ laser on chronic neck pain: a double-blind, randomized, placebo-controlled study. Pain, 124(1-2):201-210, 2006. URL https://doi.org/10.1016/j.pain.2006.05.018. [p409]

R. H. B. Christensen. ordinal-Regression Models for Ordinal Data, 2019. URL https: //CRAN. R-project. org/package=ordinal. R package version 2019.12-10. [p415]

R. H. B. Christensen, G. Cleaver, and P. B. Brockhoff. Statistical and Thurstonian models for the A-not A protocol with and without sureness. Food Quality and Preference, 22(6):542-549, 2011. URL https://doi.org/10.1016/j.foodqual.2011.03.003. [p414]

E. Demidenko. Mixed models: theory and applications with R. Wiley series in probability and statistics. Wiley, second edition, 2013. URL https://doi.org/10.1002/9781118651537. [p398]

J. W. Hardin and J. M. Hilbe. Generalized estimating equations. CRC Press, second edition, 2013. URL https://doi.org/10.1201/b13880. [p416]

T. Hothorn. Most likely transformations: The mlt package. Journal of Statistical Software, 92(1):1-68, 2020. URL https://doi.org/10.18637/jss.v092.i01. [p398, 407, 412]

T. Hothorn and L. Barbanti. tram: Transformation Models, 2021. URL https://CRAN. R-project. org/ package=tram. R package version 0.6-0. [p398]

T. Hothorn, L. Möst, and P. Bühlmann. Most likely transformations. Scandinavian Journal of Statistics, 45(1):110-134, 2018. URL https://doi.org/10.1111/sjos.12291. [p398, 400, 405, 407]

H. Joe. Accuracy of Laplace approximation for discrete response mixed models. Computational Statistics $\mathcal{E}$ Data Analysis, 52(12):5066-5074, 2008. URL https://doi.org/10.1016/j.csda.2008.05.002. [p416]

K. Kristensen, A. Nielsen, C. W. Berg, H. Skaug, and B. M. Bell. TMB: Automatic differentiation and Laplace approximation. Journal of Statistical Software, 70(5):1-21, 2016. URL https://doi . org/10. 18637/jss.v070.i05. [p400]

Y. Lee and J. A. Nelder. Conditional and Marginal Models: Another View. Statistical Science, 19(2): 219-238, 2004. URL https://doi.org/10.1214/088342304000000305. [p416]

J. K. Lindsey and P. Lambert. On the appropriateness of marginal models for repeated measurements in clinical trials. Statistics in Medicine, 17(4):447-469, 1998. URL https: //doi.org/10.1002/(SICI) 1097-0258(19980228)17:4<447: :AID-SIM752>3.0.C0;2-G. [p416]

T. Lohse, S. Rohrmann, D. Faeh, and T. Hothorn. Continuous outcome logistic regression for analyzing body mass index distributions. F1000Research, 6:1933, 2017. URL https://doi .org/10.12688/ f1000research.12934.1. [p408]

M. Manuguerra and G. Heller. Ordinal regression models for continuous scales. The International Journal of Biostatistics, 6:14-14, 2010. URL https://doi .org/10.2202/1557-4679.1230. [p408, 409]

M. Manuguerra, G. Heller, and J. Ma. Semi-parametric ordinal regression models for continuous scales. In M. Grzegorczyk and G. Ceoldo, editors, Proceedings of the 32nd International Workshop on Statistical Modelling, volume 1, pages 236-241, 2017. [p409]

M. Manuguerra, G. Z. Heller, and J. Ma. Continuous ordinal regression for analysis of visual analogue scales: The R package ordinalCont. Journal of Statistical Software, 96(8):1-24, 2020. URL https: //doi.org/10.18637/jss.v096.i08. [p409]

M. Munda, F. Rotolo, and C. Legrand. parfm: Parametric frailty models in R. Journal of Statistical Software, 51(11):1-20, 2012. URL https://doi.org/10.18637/jss.v051.i11. [p413]

J. Pinheiro and E. C. Chao. Efficient Laplacian and adaptive Gaussian quadrature algorithms for multilevel generalized linear mixed models. Journal of Computational and Graphical Statistics, 15(1): 58-81, 2006. URL https://doi.org/10.1198/106186006X96962. [p416] 
J. Pinheiro, D. Bates, S. DebRoy, D. Sarkar, and R Core Team. nlme: Linear and Nonlinear Mixed Effects Models, 2021. URL https: //CRAN. R-project. org/package=nlme. R package version 3.1-152. [p398]

V. Rondeau, Y. Mazroui, and J. R. Gonzalez. frailtypack: An R package for the analysis of correlated survival data with frailty models using penalized likelihood estimation or parametrical estimation. Journal of Statistical Software, 47(4):1-28, 2012. URL https://doi.org/10.18637/jss.v047.i04. [p413]

S. G. Self and K.-Y. Liang. Asymptotic properties of maximum likelihood estimators and likelihood ratio tests under nonstandard conditions. Journal of the American Statistical Association, 82(398): 605-610, 1987. URL https://doi.org/10.1080/01621459.1987.10478472. [p405]

B. Tamási and T. Hothorn. tramME: Transformation Models with Mixed Effects, 2021. URL https: //CRAN.R-project.org/package=tramME. R package version 0.1.2. [p398]

T. M. Therneau. coxme: Mixed Effects Cox Models, 2020. URL https://CRAN. R-project. org/package= coxme. R package version 2.2-16. [p411]

T. M. Therneau. A Package for Survival Analysis in R, 2021. URL https://CRAN.R-project.org/ package=survival. R package version 3.2-12. [p403]

J. T. Thorson and K. Kristensen. Implementing a generic method for bias correction in statistical models using random effects, with spatial and population dynamics examples. Fisheries Research, 175:66-74, 2016. URL https://doi.org/10.1016/j.fishres.2015.11.016. [p402]

Y. Tian, T. Hothorn, C. Li, F. E. Harrell Jr., and B. E. Shepherd. An empirical comparison of two novel transformation models. Statistics in Medicine, 39(5):562-576, 2020. URL https: //doi .org/10.1002/ sim. 8425. [p398]

Bálint Tamási and Torsten Hothorn

Institut für Epidemiologie, Biostatistik und Prävention

Universität Zürich

Hirschengraben 84, CH-8001 Zürich

Switzerland

ORCiD: 0000-0002-2629-7362, 0000-0001-8301-0471

balint. tamasi@uzh.ch, Torsten. Hothorn@R-project.org 